Mon. Not. R. Astron. Soc. 000,1 1-?? (2012) Printed 22 August $2018 \quad$ (MN LATEX style file v2.2)

\title{
Gas and dust from solar metallicity AGB stars
}

\author{
P. Ventura ${ }^{1}$, A. Karakas ${ }^{2}$, F. Dell'Agli³ ${ }^{3,4}$, D. A. García-Hernández ${ }^{3,4}$, \\ L. Guzman-Ramirez ${ }^{5}$ \\ ${ }^{1}$ INAF - Osservatorio Astronomico di Roma, Via Frascati 33, 00040, Monte Porzio Catone (RM), Italy \\ ${ }^{2}$ Monash Centre for Astrophysics (MoCA), School of Physics and Astronomy, Monash University, Victoria 3800, Australia \\ ${ }^{3}$ Instituto de Astrofísica de Canarias, E-38205 La Laguna, Tenerife, Spain \\ ${ }^{4}$ Departamento de Astrofísica, Universidad de La Laguna (ULL), E-38206 La Laguna, Tenerife, Spain \\ ${ }^{5}$ Leiden Observatory, Leiden University, Niels Bohrweg 2, 2333 CA Leiden, The Netherlands
}

Accepted, Received; in original form

\begin{abstract}
We study the asymptotic giant branch (AGB) evolution of stars with masses between $1 M_{\odot}-8.5 M_{\odot}$. We focus on stars with a solar chemical composition, which allows us to interpret evolved stars in the Galaxy. We present a detailed comparison with models of the same chemistry, calculated with a different evolution code and based on a different set of physical assumptions. We find that stars of mass $\geq 3.5 M_{\odot}$ experience hot bottom burning at the base of the envelope. They have AGB lifetimes shorter than $\sim 3 \times 10^{5}$ yr and eject into their surroundings gas contaminated by proton-capture nucleosynthesis, at an extent sensitive to the treatment of convection. Low mass stars with $1.5 M_{\odot} \leq M \leq 3 M_{\odot}$ become carbon stars. During the final phases the $\mathrm{C} / \mathrm{O}$ ratio grows to $\sim 3$. We find a remarkable agreement between the two codes for the low-mass models and conclude that predictions for the physical and chemical properties of these stars, and the AGB lifetime, are not that sensitive to the modelling of the AGB phase. The dust produced is also dependent on the mass: low-mass stars produce mainly solid carbon and silicon carbide dust, whereas higher mass stars produce silicates and alumina dust. Possible future observations potentially able to add more robustness to the present results are also discussed.
\end{abstract}

Key words: Stars: abundances - Stars: AGB and post-AGB - Stars: carbon

140.252.118.146

\section{INTRODUCTION}

The recent years have witnessed a growing interest in the evolution of asymptotic giant branch (AGB) stars. This is because AGB stars play an important role in various contexts of interest for the astrophysical community. In studies focused on Galaxy evolution, AGB yields are crucial for the interpretation of the chemical trends traced by stars in different parts of the Milky Way (Romano et al. 2010 Kobayashi et al. 2011). Still in the context of the Galaxy, massive AGB stars have been proposed as the main actors in the formation of multiple populations in Globular Clusters (Ventura et al. 2001, 2016b). Moving out of the Galaxy, it is generally believed that AGB stars provide an important contribution to dust production at high redshift (Valiante et al. 2009, 2011).

The research focused on AGB evolution has made significant progress in recent years. This is partly due to the improvement in computer performance, which allows faster and more exhaustive explorations of the parameter space. However, stellar evolutionary modelling is still plagued by major uncertainties in the input physics. It is now generally accepted that the treatment of convection and the description of mass loss are the two most relevant phenomena on the determination of the physical evolution of this class of objects and on the modality with which they contaminate their surroundings (for recent reviews Herwig 2005; Karakas \& Lattanzio 2014).

Some research groups have recently completed models of the AGB phase with the inclusion of dust formation processes in the wind (Nanni et al. 2013a b, 2014, Ventura et al. $2012 \mathrm{a}$ b). This is a welcome result, given that the circumstellar envelopes of AGB stars are a favourable environment for the condensation of gas molecules into solid particles (Gail \& Sedlmayr 1999). This approach is crucial for determining the type and amount of dust produced by AGB stars, and in a broader context, how they participate in the lifecycle of the Universe. This research is also necessary for interpreting the results from infrared (IR) space missions, 
Ventura et al.

considering that some of the brightest nearby objects in the IR are mass-losing dusty AGB stars.

To assess the reliability of results from the current generation of AGB models, we have recently started a research project aimed at understanding how the interpretation of the observations is sensitive to the numerical and physical input adopted to compute the evolutionary sequences. This approach, based on a comparison between results from two codes that are well known to model AGB stars and their yields, was applied to interpret and characterise the most obscured stars in the Magellanic Clouds (MC) (Ventura et al. 2015a 2016a). This choice was motivated by the fact that the research on the AGB phase has been traditionally focused on the MC instead of the Milky Way, given the largely unknown distances to the Galactic sources. The comparison was based on the metallicities $Z=4,8 \times 10^{-3}$, typical of MC stars.

We intend to apply this approach to study other environments, external to the MC. This step is of extreme importance if we consider that Gaia and the incoming launch of the James Webb Space Telescope (JWST) will definitively provide a boost in the research on AGB stars. Gaia will provide the distances to several classes of AGB stars in the Milky Way with great precision; this will allow us to overcome the major difficulty in the study of AGB stars in the Galaxy, which is their unknown distances. A lack of accurate distances prevents an exhaustive and reliable interpretation of the data. Furthermore, thanks to the JWST, we will soon have a considerable amount of IR data on resolved AGB populations in nearby galaxies, spanning a range of mean metallicities (Jones et al. 2017).

In order to be prepared for these upcoming observational challenges, it is important to fix the critical and most uncertain points in the description of the AGB evolution and to select the results for which different studies reach similar conclusions. To this aim, here we provide a step forward by extending the analysis done by Ventura et al. (2015a) and Ventura et al. (2016a) to stars of solar metallicity. We compare the results published by Karakas (2014) and Karakas \& Lugaro (2016), calculated with the MONASH code, with new, updated models of solar metallicity, calculated with the ATON code. These ATON models have been calculated with the same metallicity $(Z=0.014)$ and the same mixture (Asplund et al.2009) adopted by Karakas (2014), to allow a straightforward comparison. The analysis will be focused on the physical properties of stars of different progenitor mass, on the chemistry of the gas expelled into the circumstellar environment, and on the dust produced. The comparison with the recent explorations by Di Criscienzo et al. (2016) and Dell'Agli et al. (2017, hereinafter D17), based on the solar mixture by Grevesse \& Sauval (1998), will be used to assess the differences due to the particular solar mixture adopted.

The paper is structured as follows. The description of the input physics used to build the evolutionary sequences and to model dust formation are given in Sections 2 and 3 , respectively. In Section 4 we present an overview of the main physical phenomena affecting the description of the AGB phase, while the physical and chemical properties of the AGB stars presented here are discussed in Sections 5 and 6 , respectively. Section 7 presents the final chemical composition, a summary of the observational limitations regard- ing present chemical abundances determinations in Galactic AGB stars and their descendants such as post-AGB stars and planetary nebulae $(\mathrm{PNe})$ as well as some future observational directions that would be useful to test the theoretical AGB models. The gas and dust yields are presented in Section 8 and 9, respectively, while in Section 10 we discuss the metallicity effects on the evolutionary properties of AGB stars. Finally, the conclusions are given in Section 11.

\section{STELLAR MODELLING}

The models presented in this work were calculated with the ATON code (Ventura et al. 1998). Each model was evolved from the main sequence until the almost total consumption of the external mantle. The results will be compared with models by Karakas (2014), calculated with the MONASH version of the Mount Stromlo Stellar Structure Program (Frost \& Lattanzio 1996). In the following we will refer to the two sets of models as ATON and MONASH models, respectively. An exhaustive description of the numerical details of the codes (along with the most recent updates) can be found in Ventura et al. (2013) and in Karakas (2014). Here we proviede a brief summary of the physical input most relevant to this work and outline the differences between ATON and MONASH.

\section{$2.1 \quad$ Initial chemistry}

The models calculated span the mass interval $1 M_{\odot} \leq M \leq$ $8.5 M_{\odot}$. The metallicity used is $Z=0.014$ and the mixture adopted is taken from Asplund et al. (2009). The initial helium is $Y=0.265$ in the ATON case, whereas the MONASH models are computed with $Y=0.28$. This difference in the initial helium has some effects on the extent of the third dgredge-up (hereinafter TDU), which is more efficient the lower is the helium in the star.

\subsection{Convection}

In the ATON case the temperature gradient within regions unstable to convection is found via the Full Spectrum of Turbulence (FST) model (Canuto \& Mazzitelli 1991). The MONASH sequences are based on the Mixing Length Theory (MLT), with the mixing length parameter $\alpha=1.86$. For the determination of the extension of the mixing region, in the ATON case it is assumed that the velocity of convective eddies decay exponentially beyond the neutrality point, fixed via the Schwartzschild criterion: the e-folding distance of the velocity decays during the core (hydrogen and helium) burning phases and during the AGB phase is taken as $0.02 H_{P}$ and $0.002 H_{P}$, respectively.

In the MONASH model we apply the algorithm described by Lattanzio (1986) in order to search for convective neutrality at the border between all radiative and convective regions. This method has been shown to increase the depth of TDU relative to schemes that apply the Schwartzschild criterion (e.g., Frost \& Lattanzio 1996). However Kamath et al. (2012) showed that this algorithm does not provide enough TDU at an small enough core mass to match the observations of AGB stars in Magellanic Cloud Clusters. Some overshoot is needed, especially at the lowest masses 
to experience it. For this reason, a simple overshoot scheme is applied to the base of the convective envelope during the AGB in models of mass $M=1.5,1.75 \mathrm{M}_{\odot}$ to allow these cases to become carbon rich (we refer to Karakas \& Lugaro 2016, for details). No overshoot is however used in models with $M \geq 2 \mathrm{M}_{\odot}$.

\subsection{Mass loss}

The mass loss rate for oxygen-rich ATON models is determined via the Blöcker (1995) treatment; the parameter entering the Blöcker (1995)'s recipe is set to $\eta=0.02$, following the calibration given in Ventura et al. (2000). For carbon stars the ATON calculations are based on the description of mass loss from the Berlin group (Wachter et al. 2002 2008). In the MONASH case the mass-loss formulation by Vassiliadis \& Wood (1993) is adopted.

\subsection{Opacities}

In both codes the radiative opacities are calculated according to the OPAL release, in the version documented by Iglesias \& Rogers (1996). The molecular opacities in the lowtemperature regime $\left(T<10^{4} \mathrm{~K}\right)$ are calculated by means of the AESOPUS tool (Marigo \& Aringer 2009). The opacities are suitably constructed to follow the changes in the chemical composition of the envelope, particularly of the individual abundances of carbon, nitrogen, and oxygen.

\section{THE DESCRIPTION OF DUST FORMATION}

The description of dust formation is based on the pioneering formalism introduced by the Heidelberg group (Ferrarotti \& Gail 2001, 2002, 2006). The full set of equations, with an exhaustive discussion of the role played by the different physical quantities, can be found in previous papers (Ventura et al. 2012a b Di Criscienzo et al. 2013).

The model is based on the assumption that the outflow expands symmetrically from the surface of the star and that dust formation occurs within the condensation zone, where the temperatures are sufficiently low that the rate of growth of dust grains overcomes the rate of vaporisation.

On the mathematical side we consider two independent variables, namely the velocity of the gas and the optical depth, whose behaviour is described by two differential equations.

The first equation is the expression of momentum conservation: the acceleration of gas particles is given by the balance between gravity and radiation pressure.

$$
v \frac{d v}{d r}=-\frac{G M_{*}}{r^{2}}(1-\Gamma),
$$

where $\Gamma$ represents the effects of radiation pressure on dust particles. When $\Gamma$ is above unity the wind is accelerated. The expression for $\Gamma$ is the following:

$$
\Gamma=\frac{k L_{*}}{4 \pi c G M_{*}},
$$

where $k, M_{*}$ and $L_{*}$ indicate, respectively, the extinction coefficient, the mass and the luminosity of the star.
The equation for the optical depth is the following:

$$
\frac{d \tau}{d r}=-k \rho\left(\frac{R_{*}}{r}\right)^{2},
$$

where $\rho$ is the density of the gas.

The two above equations are completed by the mass conservation equation, for density, and the relationship giving the radial variation of temperature as a function of the effective temperature of the star:

$$
\begin{gathered}
\rho=\frac{\dot{M}}{4 \pi r^{2} v}, \\
T^{4}=\frac{1}{2} T_{\text {eff }}^{4}\left[1-\sqrt{1-\left(\frac{R_{*}}{r}\right)^{2}}+\frac{3}{2} \tau\right] .
\end{gathered}
$$

The growth of dust grains is given by the difference between the rate of the addition of gas molecules on preexisting solid particles and the vaporisation rate. This requires the introduction of additional differential equations, one for each dust species considered.

The choice of the dust species is based on the argument of molecular stability. The most relevant assumption is the stability of the $\mathrm{CO}$ molecule, which absorbs entirely into $\mathrm{CO}$ molecules the least abundant between $\mathrm{C}$ and $\mathrm{O}$.

In oxygen-rich environments we consider the formation of alumina dust $\left(\mathrm{Al}_{2} \mathrm{O}_{3}\right)$, silicates and solid iron. The key elements for the formation of these dust species are aluminium, silicon and iron. For carbon stars we follow the formation of solid carbon grains, silicon carbide and solid iron; in this case the key elements are carbon, silicon and iron.

\section{THE KEY FACTORS AFFECTING AGB EVOLUTION MODELLING}

Fig. 1 shows the core mass $M_{\mathrm{c}}$ at the beginning of the TPAGB phase for the models discussed in the present work. This quantity is reported into col. 5 of Table 1 . We show for comparison the results from Karakas (2014) and Karakas \& Lugaro (2016). In the mass domain $M \leq 5 M_{\odot}$ the results are very similar. Conversely, for $M>5 M_{\odot}$ the results diverge, with the ATON models reaching the TP-AGB phase with a more massive core. The largest difference of $\sim 0.2 M_{\odot}$ is reached for $M=8 M_{\odot}$.

The core-mass threshold for hot bottom burning (HBB) is $\sim 0.7 M_{\odot}$ in the ATON code (Ventura et al. 2013), which is lower than in the MONASH code, where the threshold is $\gtrsim 0.85 \mathrm{M}_{\odot}$. The ignition of HBB has an important effect on the luminosity evolution of the star (Renzini \& Voli 1981 Blöcker \& Schöenberner 1991), and on the surface chemical composition.

Before entering the general discussion of the properties of AGB stars of solar metallicity, we present the main features of the evolution of stars undergoing $\mathrm{HBB}$ and their lower mass counterparts. We select the $5 \mathrm{M}_{\odot}$ and the $3 \mathrm{M}_{\odot}$ models from the ATON and MONASH codes as being representative of stars with $\mathrm{HBB}$ and stars that become carbon rich. As shown in Fig. 1 the core masses at the beginning of the TP-AGB phase are very similar in the ATON and 
Table 1. Physical properties of solar metallicity AGB models.

\begin{tabular}{cccccccccc}
\hline \hline$M / \mathrm{M}_{\odot}$ & $\tau_{M S}$ & $\tau_{A G B}$ & $\tau_{T P-A G B}$ & $M_{c} / \mathrm{M}_{\odot}^{a}$ & $L_{\max } / L_{\odot}$ & $T_{b c e}^{\max }(M K)$ & $N_{T P}$ & $\lambda$ & $M_{f} / \mathrm{M}_{\odot}$ \\
\hline 1.00 & $1.0 \mathrm{E}+10$ & $2.92 \mathrm{E}+07$ & $9.12 \mathrm{E}+05$ & 0.515 & $3.80 \mathrm{E}+03$ & 1 & 5 & 0.04 & 0.534 \\
1.25 & $4.4 \mathrm{E}+09$ & $2.73 \mathrm{E}+07$ & $1.63 \mathrm{E}+06$ & 0.523 & $6.53 \mathrm{E}+03$ & 3 & 11 & 0.05 & 0.589 \\
1.50 & $2.6 \mathrm{E}+09$ & $2.67 \mathrm{E}+07$ & $1.85 \mathrm{E}+06$ & 0.523 & $8.41 \mathrm{E}+03$ & 4 & 16 & 0.25 & 0.618 \\
1.75 & $1.7 \mathrm{E}+09$ & $2.79 \mathrm{E}+07$ & $2.22 \mathrm{E}+06$ & 0.527 & $1.00 \mathrm{E}+04$ & 6 & 19 & 0.34 & 0.636 \\
2.00 & $1.2 \mathrm{E}+09$ & $3.27 \mathrm{E}+07$ & $2.74 \mathrm{E}+06$ & 0.516 & $1.05 \mathrm{E}+04$ & 7 & 23 & 0.31 & 0.646 \\
2.25 & $8.4 \mathrm{E}+08$ & $3.15 \mathrm{E}+07$ & $4.25 \mathrm{E}+06$ & 0.488 & $1.23 \mathrm{E}+04$ & 10 & 34 & 0.37 & 0.673 \\
2.50 & $6.3 \mathrm{E}+08$ & $2.42 \mathrm{E}+07$ & $3.52 \mathrm{E}+06$ & 0.500 & $1.32 \mathrm{E}+04$ & 13 & 35 & 0.40 & 0.669 \\
2.75 & $4.8 \mathrm{E}+08$ & $1.76 \mathrm{E}+07$ & $2.75 \mathrm{E}+06$ & 0.526 & $1.43 \mathrm{E}+04$ & 18 & 35 & 0.40 & 0.699 \\
3.00 & $3.9 \mathrm{E}+08$ & $1.25 \mathrm{E}+07$ & $1.91 \mathrm{E}+06$ & 0.565 & $1.53 \mathrm{E}+04$ & 22 & 31 & 0.43 & 0.709 \\
3.50 & $2.6 \mathrm{E}+08$ & $6.00 \mathrm{E}+06$ & $9.16 \mathrm{E}+05$ & 0.670 & $2.34 \mathrm{E}+04$ & 67 & 41 & 0.28 & 0.822 \\
4.00 & $1.7 \mathrm{E}+08$ & $3.10 \mathrm{E}+06$ & $3.15 \mathrm{E}+05$ & 0.793 & $3.16 \mathrm{E}+04$ & 84 & 36 & 0.27 & 0.875 \\
4.50 & $1.4 \mathrm{E}+08$ & $1.98 \mathrm{E}+06$ & $2.26 \mathrm{E}+05$ & 0.834 & $3.89 \mathrm{E}+04$ & 86 & 35 & 0.13 & 0.903 \\
5.00 & $1.1 \mathrm{E}+08$ & $1.35 \mathrm{E}+06$ & $2.11 \mathrm{E}+05$ & 0.864 & $4.68 \mathrm{E}+04$ & 90 & 33 & 0.12 & 0.935 \\
5.50 & $8.5 \mathrm{E}+07$ & $9.31 \mathrm{E}+05$ & $1.31 \mathrm{E}+05$ & 0.899 & $5.50 \mathrm{E}+04$ & 91 & 31 & 0.10 & 0.955 \\
6.00 & $7.0 \mathrm{E}+07$ & $6.64 \mathrm{E}+05$ & $7.78 \mathrm{E}+04$ & 0.938 & $6.46 \mathrm{E}+04$ & 93 & 28 & 0.10 & 0.980 \\
6.50 & $5.9 \mathrm{E}+07$ & $4.76 \mathrm{E}+05$ & $4.86 \mathrm{E}+04$ & 0.986 & $7.76 \mathrm{E}+04$ & 96 & 26 & 0.10 & 1.020 \\
7.00 & $5.0 \mathrm{E}+07$ & $3.47 \mathrm{E}+05$ & $4.32 \mathrm{E}+04$ & 1.045 & $8.91 \mathrm{E}+04$ & 99 & 26 & 0.10 & 1.084 \\
7.50 & $4.4 \mathrm{E}+07$ & $2.88 \mathrm{E}+05$ & $5.30 \mathrm{E}+04$ & 1.110 & $9.33 \mathrm{E}+04$ & 100 & 26 & 0.08 & 1.141 \\
8.00 & $3.8 \mathrm{E}+07$ & $2.26 \mathrm{E}+05$ & $4.19 \mathrm{E}+04$ & 1.230 & $1.07 \mathrm{E}+05$ & 104 & 24 & 0.05 & 1.248 \\
8.50 & $3.4 \mathrm{E}+07$ & $1.78 \mathrm{E}+05$ & $2.80 \mathrm{E}+04$ & 1.310 & $1.29 \mathrm{E}+05$ & 118 & 21 & 0.03 & 1.315 \\
\hline
\end{tabular}

${ }^{a}$ Core mass at the beginning of the TP-AGB phase.

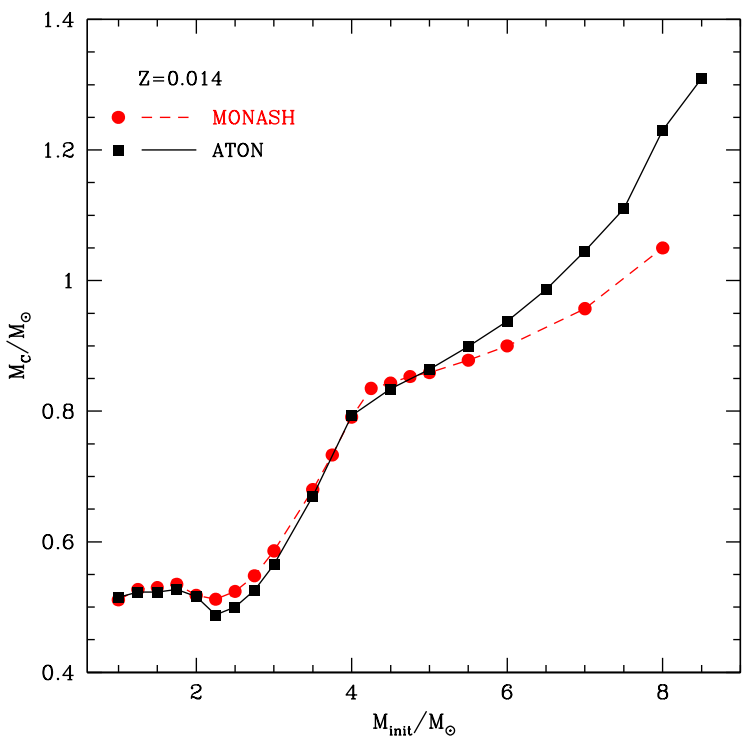

Figure 1. The core mass at the beginning of the TP-AGB phase for the solar metallicity models presented here. Full, black squares connected with a solid line, indicate the results obtained with the ATON code, whereas the red points, connected by a dashed line, indicate the results published in Karakas (2014) and Karakas \& Lugaro (2016), obtained with the MONASH code.

MONASH models: this will allow us to disentangle the effects of the various physics input adopted, without taking care of possibile differences arising from the pre-TP-AGB phase.

\subsection{The evolution of massive AGB stars: the role of HBB}

To understand the main features of the evolution of stars experiencing $\mathrm{HBB}$ we show in Fig. 2 the variation of the luminosity and of the core mass for a $5 \mathrm{M}_{\odot}$ model, compared with the corresponding model by Karakas (2014).

We see that the maximum luminosity reached and the overall duration of the AGB phase differ. The ATON model reaches a luminosity $L_{\max } \sim 4.5 \times 10^{4} L_{\odot}$ significantly higher than MONASH $\left(L_{\max } \sim 3 \times 10^{4} L_{\odot}\right)$. This is a direct result of the FST model of convection in the ATON case: as shown by Ventura \& D'Antona (2005), FST models experience a stronger HBB and evolve at larger luminosities in comparison with models calculated with the MLT.

The difference in the luminosity in turn affects the overall duration of the AGB phase. Owing to the larger luminosities, the ATON model is exposed to larger rates of mass loss, thus the envelope is lost faster and the duration of this phase is shorter. This is clearly shown in both panels of Fig. 2. where we see that in the MONASH model the envelope is lost in $\sim 0.4 \mathrm{Myr}$, approximately double the evolution time of the FST model.

The present results confirm the analysis by Ventura et al. (2015a), which outlined the effects of convection modelling on the luminosity and the duration of AGB models experiencing HBB, with metallicities typical of LMC stars.

The description of convective zones affects the luminosity and consequently the growth rate of the core, $\dot{M}_{\mathrm{C}}$, because the luminosity determines the rapidity with which the CNO-burning shell moves outwards (in mass). This is confirmed by the results shown in the right panel of Fig. 2 , indicating that $\dot{M}_{\mathrm{C}}$ is higher in the ATON case. A direct consequence of the higher core-mass growth is the final mass of the star, which is larger in the ATON case. We will go back to this point in section 5 

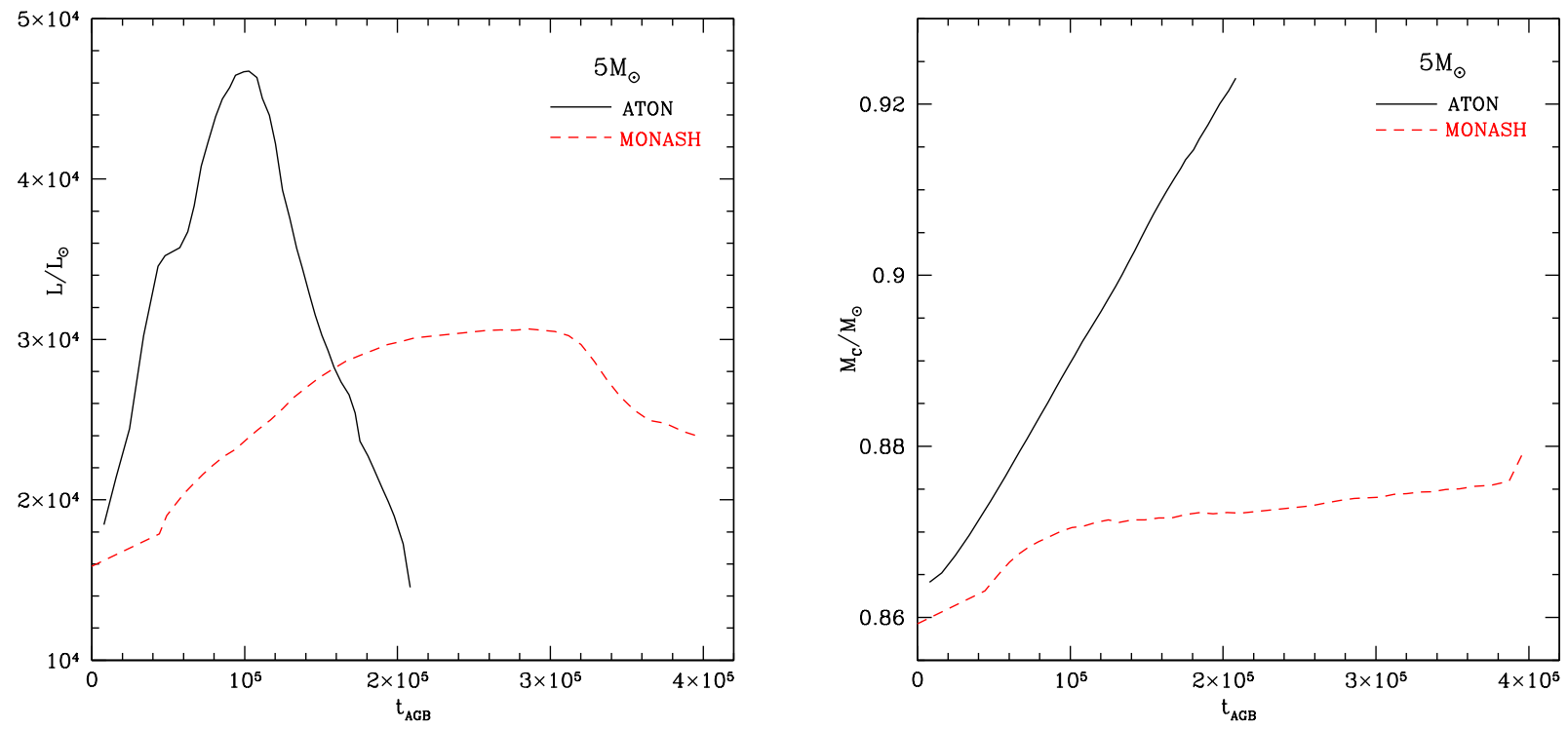

Figure 2. The variation with time (counted from the beginning of the TP-AGB phase) of luminosity (left panel) and core mass (right) for $5 \mathrm{M}_{\odot}$ stars. The results from ATON are shown with a black, solid line, whereas the red, dashed line indicates the results from MONASH code.
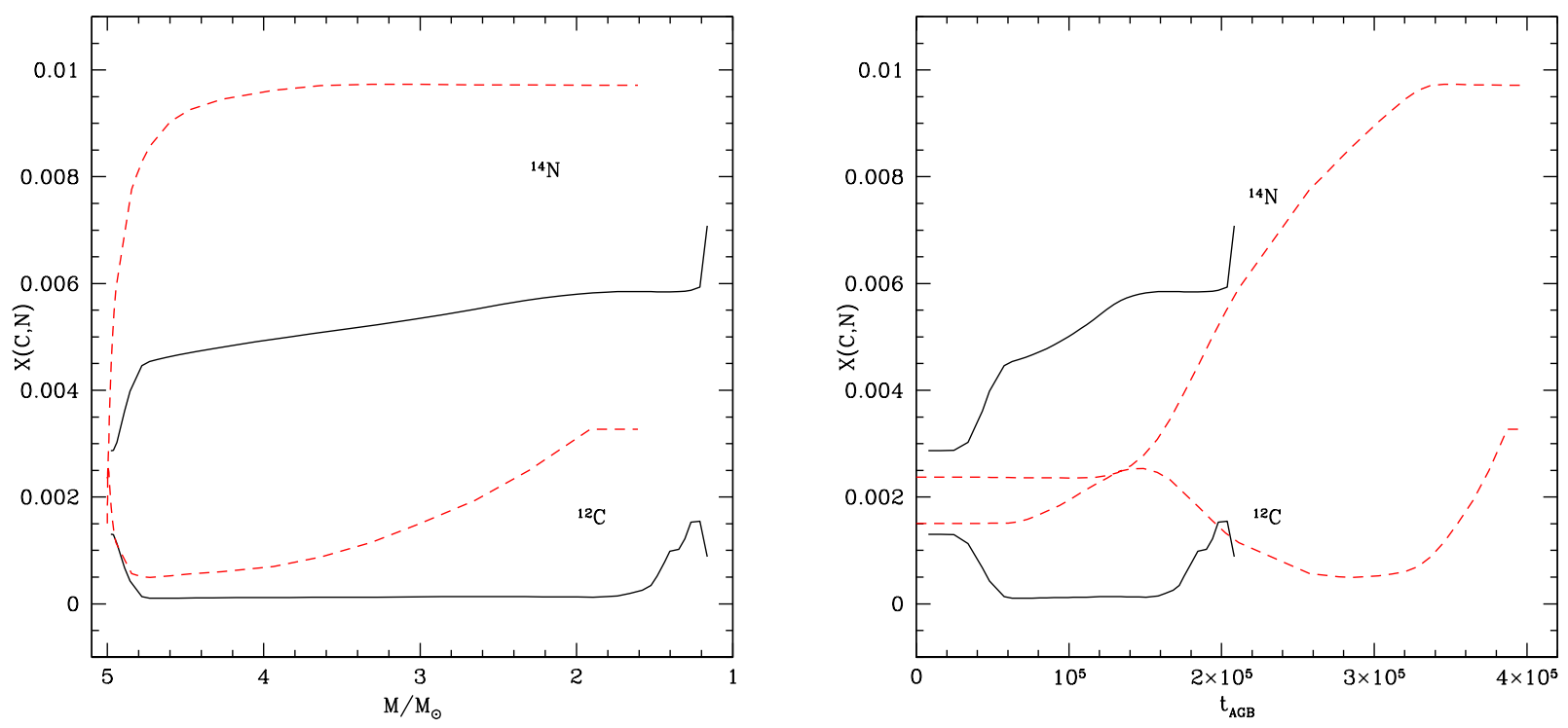

Figure 3. The variation of the surface abundances of carbon and nitrogen during the AGB phase for the same models shown in Fig. 2 calculated with the ATON (black, solid line) and MONASH (red, dashed track) codes. In the left panel in the abscissa we show the current mass of the star, whereas in the left panel we show the time, counted from the beginning of the TP-AGB phase.

The differences in the evolution of the main physical properties of massive AGB stars affect the variation of the surface chemical composition. Fig. 3 shows the evolution of the surface mass fraction of carbon and nitrogen. In the left panel we show the current mass of the star on the abscissa, to have an idea of the contamination of the interstel- lar medium from these objects. Generally speaking, we find nitrogen production, a clear signature of the activation of HBB. The main difference we observe is in the behaviour of carbon. This is because in the ATON models carbon is destroyed from the very first thermal pusles (hereinafter TP) and is found to be a factor $\sim 20$ lower than the initial value 


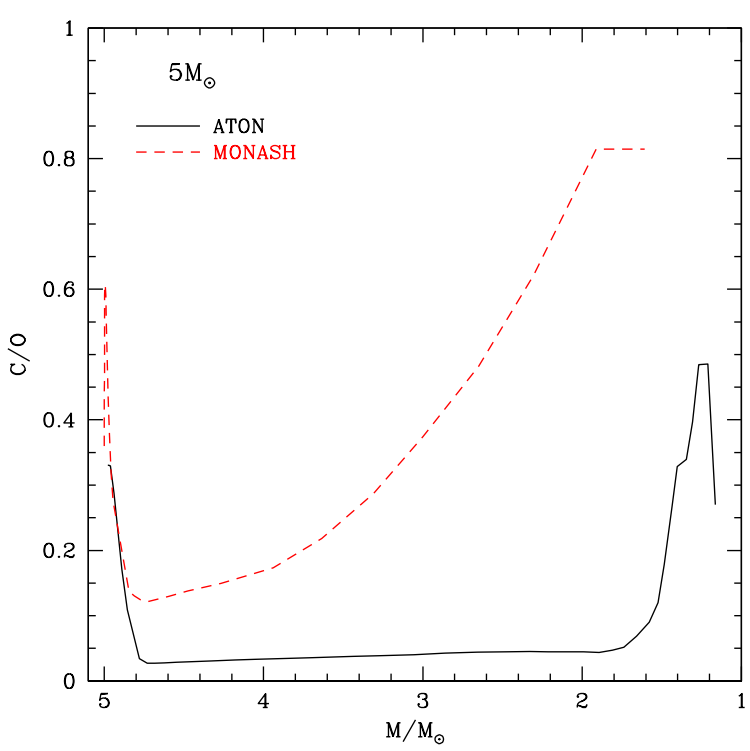

Figure 4. The variation of the surface $\mathrm{C} / \mathrm{O}$ ratio in the same models shown in Fig. 2

for most of the AGB phase. Only in the very final evolutionary stages do we see some carbon transported to the surface by TDU. It is clear from the left panel of Fig. 3 that the ejecta from this star is carbon poor.

When the MLT model for convection is used, the situation is considerably different. We note that the surface carbon first increases owing to the action of TDU during the first few TPs before HBB is activated. Furthermore, the destruction of carbon is milder during the total AGB phase, and in the final evolutionary stages the surface carbon abundance grows to be larger than at the beginning of the AGB phase. The net yield is negative where the average $\mathrm{C}$ mass fraction in the wind is $\approx 60 \%$ lower than the initial.

In Fig 4 we show the evolution of the surface $\mathrm{C} / \mathrm{O}$ ratio, which we will see is important for a number of issues and is also deeply affected by convection modelling. In the ATON case, the strong HBB conditions ensures that model evolves with $\mathrm{C} / \mathrm{O}<0.05$ for most of the AGB phase. In the MONASH model, after an initial phase of decrease, the surface $\mathrm{C} / \mathrm{O}$ gradually increases until reaching $\mathrm{C} / \mathrm{O} \sim 0.8$ towards the end of the evolution.

A general result outlined by these models is the synthesis of nitrogen. In the MONASH case the quantity of nitrogen synthesized is higher, because of a dominant primary component, produced by proton captures on carbon nuclei synthesized in the convective shell formed during each TP and convected to the envelope via TDU. In the ATON case, because TDU has only modest effects, the secondary component is dominant in this range of mass.

If the temperatures exceed $\sim 80 \mathrm{MK}$ oxygen can be destroyed via proton captures, while the sodium and aluminium may be produced (Izzard et al. 2007). It is generally recognized that this occurs in Pop II, massive AGB stars, given the large HBB temperatures experienced (Ventura et al. 2013, Fishlock et al. 2014). To check whether this ad- vanced nucleosynthesis occurs at solar metallicites, we show in Fig. 5 the production factors of oxygen, sodium and aluminium for the $5 M_{\odot}$ models presented in Fig. 2, 3 and 4 calculated with ATON (left) and MONASH (right). We also show, for completeness, the evolution of the surface lithium, which will be discussed in more details in section 6.3.

The depletion of the surface oxygen is higher in the ATON case, owing to the stronger HBB conditions; however, the overall oxygen destruction is below $\sim 0.2$ dex: the gas ejected by these stars, independently of the description of convection used, is characterized by only a modest depletion in the oxygen content. For what attains sodium, in the ATON model we find a significant production, almost by a factor $\sim 4$, a signature of the activation of ${ }^{22} \mathrm{Ne}+p$ at the base of the envelope; accordingly, the gas ejected by these stars is expected to be sodium-rich; in the MONASH case a much smaller increase in the sodium content is found. The difference in the behaviour of sodium is due to the combined effects of convection modelling and of the crosssection adopted; the ATON models have been calculated by assuming the upper limits given by Hale et al. (2002) for the ${ }^{22} \mathrm{Ne}+p$ reaction rates, wheres the MONASH results are based on the recommended values. Finally, we see in Fig. 5 that only a modest production of aluminium is expected, consistently with the low efficiency of $\mathrm{HBB}$ at solar metallicities.

\subsection{Low mass stars and the C-star phase}

Stars of mass $\lesssim 4 M_{\odot}$ do not experience HBB and their surface chemistry is affected only by TDU episodes, which may eventually turn the star into a carbon star.

The top panels of Fig. 6 shows the evolution of the luminosity and effective temperature of the $3 M_{\odot}$ models. The luminosity increases steadily during the AGB phase, from $\sim 5 \times 10^{3} L_{\odot}$ to $\sim 1.5 \times 10^{4} L_{\odot}$. At the same time the effective temperature decreases as the star expands, starting from $T_{\text {eff }} \sim 4000$ at the beginning of the TP-AGB evolution. The cooling of the external regions of the star are particularly important after the C-star stage is reached: this is caused by the significant increase in the molecular opacities in carbon-rich gas (Marigo 2002, Ventura \& Marigo 2009 2010).

When comparing the present results with Karakas (2014) we note that, unlike more massive stars, the luminosities are independent of convective modelling. This is because no HBB is experienced, which means no contribution from the internal regions of the envelope to the overall energy release.

The evolution of the effective temperature is illustrated in the right, top panel of Fig. 6. Here we see some similarities but also important differences between the ATON and MONASH results, suggesting that the treatment of convection may have some effect here. For $\sim 90 \%$ of the AGB phase the effective temperatures are rather similar, with $T_{\text {eff }}$ decreasing from $\sim 4000 \mathrm{~K}$ to $T_{\text {eff }} \sim 3000 \mathrm{~K}$.

When the surface $\mathrm{C} / \mathrm{O}$ overcomes $\sim 1.4$ the ATON

1 We define the production factor of a given element as the ratio between the surface mass fraction of that element at a given time and the initial abundance, with which the star formed. 

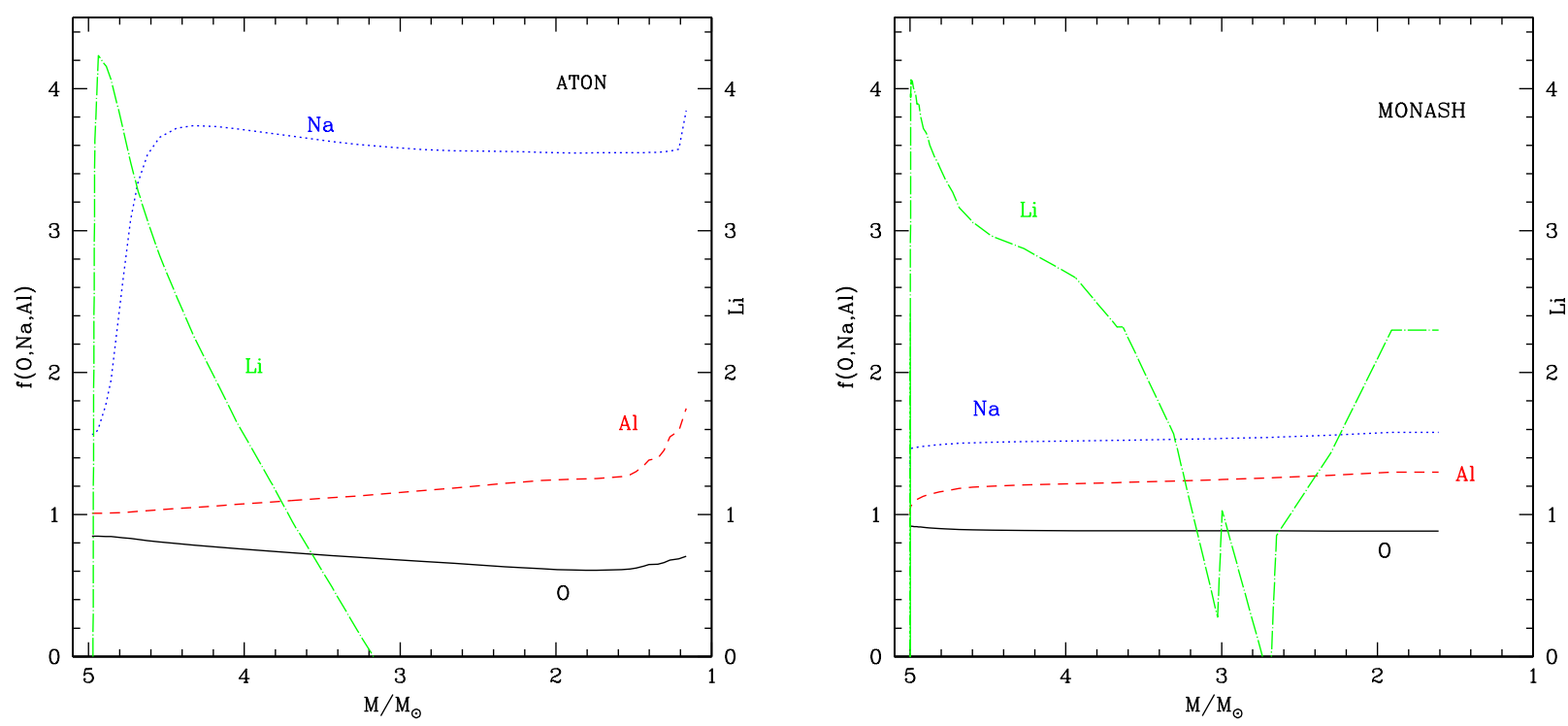

Figure 5. The evolution of the production factors of oxygen (black, solid line), sodium (blue, dotted), aluminium (red, dashed) during the AGB phase of a $5 M_{\odot}$ model calculated with the ATON (left panel) and MONASH (right) codes. The green, dotted-dashed lines indicates the surface abundance of lithium, in the standard units, $\log \epsilon\left({ }^{7} L i\right)=12+\log \left(n\left({ }^{7} L i\right) / n(H)\right)$ (scale on the right).

temperatures become extremely cool, until reaching $T_{\text {eff }} \sim$ $2000 \mathrm{~K}$ in the very final evolutionary stages. In the model by Karakas (2014) the effective temperature is above $2500 \mathrm{~K}$ for the whole AGB evolution.

This dissimilarity is due to the development of a region within the envelope where the convective efficiency, $\Sigma$ is extremely smal ${ }^{2}$ of the order of $\Sigma \sim 0.05$. In these conditions, the ratio between the convective flux $(\Phi)$ found via the FST model and the MLT flux is $\Phi_{F S T} / \Phi_{M L T} \sim 0.1$ (see Fig. 5 in Canuto \& Mazzitelli 1991), which implies that the FST description requires an overadiabaticity peak narrower and higher than MLT. Indeed we find in the FST case $\nabla-\nabla_{a d} \sim 10$, whereas in the MLT case we would find $\nabla-\nabla_{a d} \sim 1$ for the same physical conditions. This dissimilarity in the overadiabaticity peak is the reason for the difference in the effective temperatures. This is the first time within the context of AGB modelling that we encounter a situation where the treatment of convection has an impact on the temperature gradient within the outermost regions of the star.

The smaller effective temperatures favour larger rates of mass loss, thus shorter time scales, independently of the mass loss description. While these differences are within $\sim$ $10 \%$, we will see that this will have an important impact on the production of dust by these stars.

A general result found here is that the carbon-star phase is shorter than the oxygen-rich phase, accounting for only $\sim$ $15 \%$ of the total AGB evolution. For this reason the chance of detecting these stars during the initial O-rich phase is

2 In the present work we use the same definition of the convective efficiency adopted by Canuto \& Mazzitelli (1991), given in their Eq. 5 . higher. On the other hand, the gas ejected by these stars is carbon rich. This can be understood by looking at the right, bottom panel of Fig. 6. which shows the evolution of the surface $\mathrm{C} / \mathrm{O}$ as a function of the (current) mass of the star. We see that most of the mass expelled is carbon-rich, which therefore means that the yields will also be similarly carbon rich (e.g., Cristallo et al. 2015, Karakas \& Lugaro 2016). This is due to the fact that most of mass loss occurs after the carbon-star stage is reached. In the ATON case the $\mathrm{C} / \mathrm{O}$ reached is smaller compared to MONASH, because the fast mass loss occurring in the final AGB phase prevents additional TDU events.

\section{PHYSICAL PROPERTIES OF AGB STARS}

Table 1 reports important physical quantities of the AGB models presented here, which includes the duration of the main sequence, AGB and TP-AGB phases, the core mass at the beginning of the AGB (we have discussed this quantity when analyzing Fig. 1), the maximum luminosity, the maximum temperature at the base of the envelope, the number of thermal pulses experienced, the maximum TDU parameter, $\lambda$, and the final mass of the star.

\subsection{The brightness of AGB stars}

Fig. 7 shows the maximum luminosity $\left(L_{\max }\right)$ reached during the TP-AGB evolution as a function of the initial mass $\left(M_{\text {init }}\right)$. Stars not experiencing HBB evolve at luminosities below $1.5 \times 10^{4} L \odot$. The sudden change in the slope of the $L_{\max }$ vs $M_{\text {init }}$ relationship occurring at $\sim 3.5 M_{\odot}$ is because stars experiencing HBB deviate from Paczyński (1970)'s 

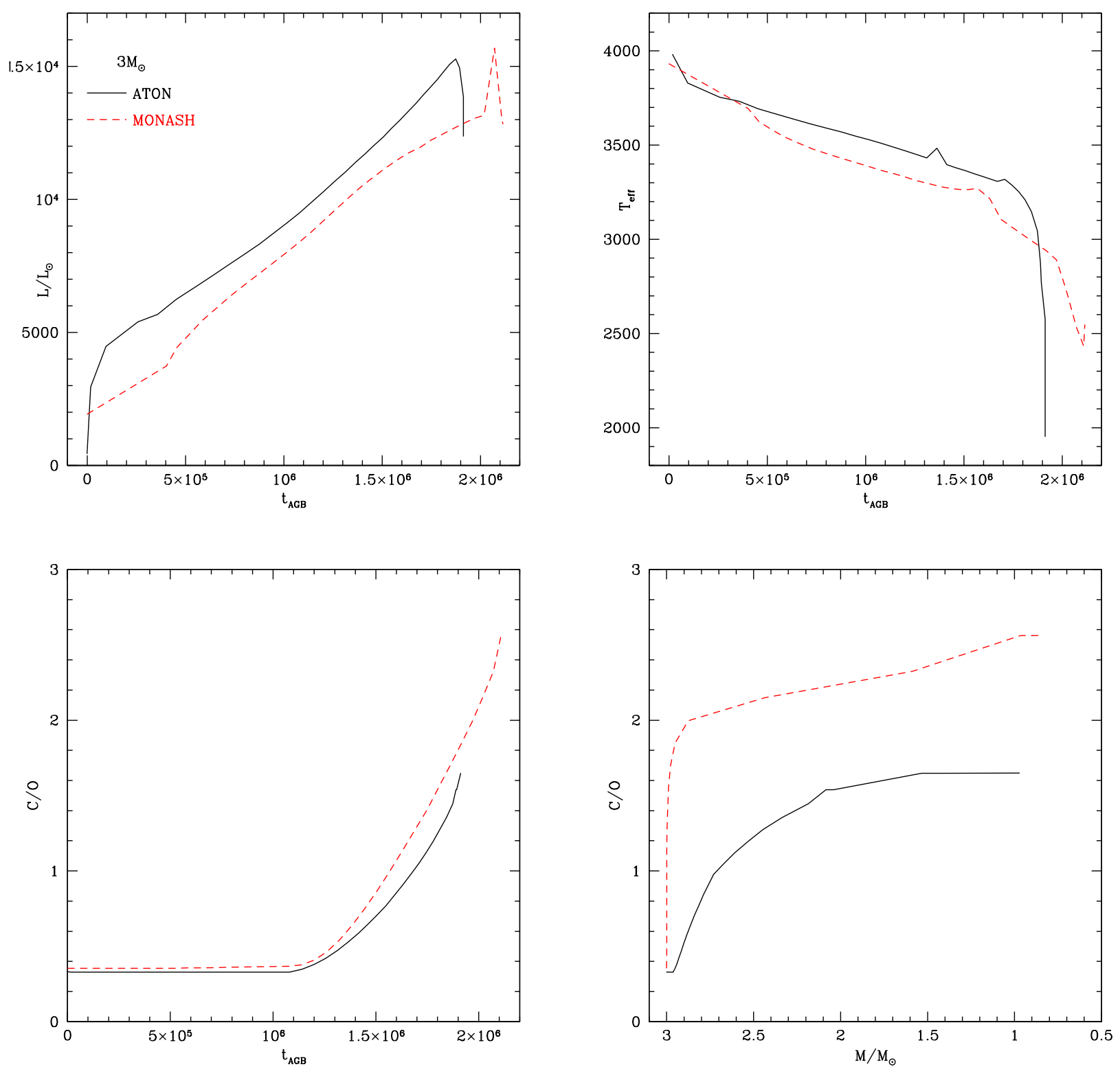

Figure 6. The variation with time of the luminosity (left, top panel), effective temperature (right, top), surface C/O (left, bottom) for two models of initial mass $3 M_{\odot}$ calculated with the ATON (black, solid track) and MONASH (red, dotted) codes. The evolution of the surface $\mathrm{C} / \mathrm{O}$ is also shown as a function of the current mass of the star in the bottom-right panel.

core mass - luminosity law (Blöcker \& Schöenberner 1991): in this mass domain we find $2 \times 10^{4} L_{\odot}<L<1.2 \times 10^{5} L_{\odot}$.

\subsection{The evolutionary time scale}

The duration of the TP-AGB phase, $\tau_{T P-A G B}$, is shown in Fig. 8. Low-mass AGB stars evolve on time scales above $\sim 1$ Myr. In this mass range the evolutionary time scale is determined by two factors, which have opposite effects on $\tau_{T P-A G B}$. The mass of the envelope (higher masses require longer times to be lost) and the luminosity (which, as shown in Fig, 7, increases with the mass of the star). This is the reason why the trend with mass is not monotonic. The stars with the longest TP-AGB evolution, of the order of $\sim 5$ Myr, are those with $M_{\text {init }} \sim 2 M_{\odot}$.

For stars experiencing HBB the time scale of the TPAGB evolution is determined essentially by the luminosity. $\tau_{T P-A G B}$ decreases with $M_{\text {init }}$, because higher mass models have larger luminosities. The $8.5 M_{\odot}$ is the fastest evolving model, with a TP-AGB duration of only $\sim 3 \times 10^{4} \mathrm{yr}$. 


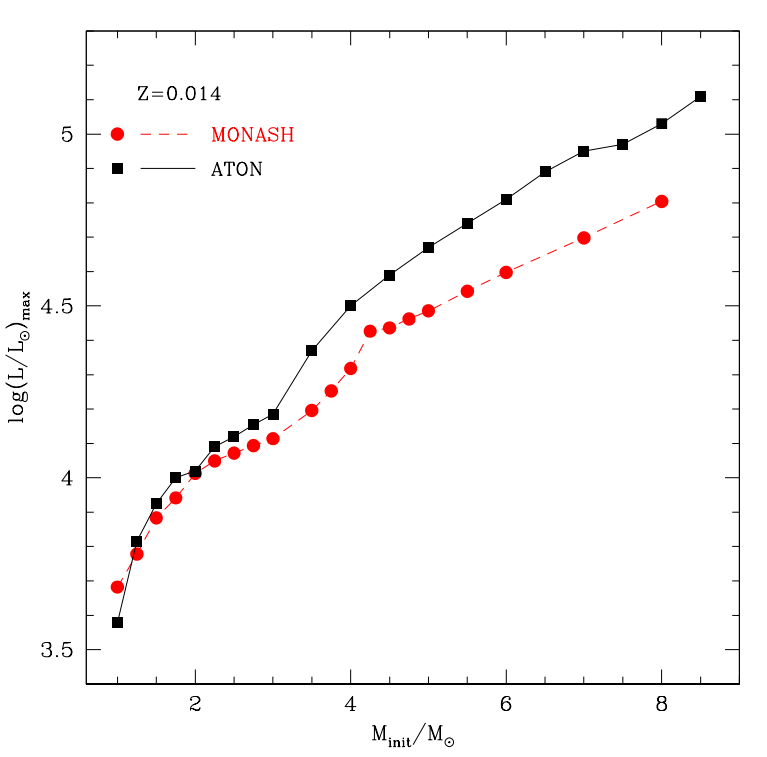

Figure 7. The maximum luminosity reached during the TP-AGB phase by stars of different mass is shown with black squares, connected by a solid line. For comparison we also show the results by Karakas (2014), indicated with red circles, connected with a dashed line.

\subsection{The initial-final mass relationship}

Fig. 9 shows the initial-final mass relationship. The mass of the envelope and the luminosity affect the final mass of the star, hence the mass of the remnant. The pre-AGB evolution is also important for the final mass of the star, because the initial mass of the core at the beginning of the TP-AGB phase depends on $M_{\text {init }}$, as shown in Fig. 1 .

Stars of initial mass $M_{\text {init }} \leq 3 M_{\odot}$ develop core masses in the range $0.55 M_{\odot}-0.7 M_{\odot}$; the final mass increases with $M_{\text {init }}$, for almost the whole range of masses involved. Turning to the stars experiencing HBB the results shown in Fig. 9 outline a sudden rise in the final core mass, which increases from $0.7 M_{\odot}$ (for $3 M_{\odot}$ stars) to $0.85 M_{\odot}\left(3.5 M_{\odot}\right.$ stars). For the stars in the range $3.5 M_{\odot}<M_{\text {init }}<8.5 M_{\odot}$, the final core mass increases monotonically, from $0.85 M_{\odot}$ to $\sim 1.3 M_{\odot}$.

In Fig. 9 we show the results from Kalirai et al. (2014), where the authors report the analysis of White Dwarfs in the clusters Hyades, Praesepe, NGC 6819 and NGC 7789. From their analysis, an initial-final mass relation was determined for low and intermediate mass stars in the initial mass range $M_{\text {init }} \leq 4 M_{\odot}$. The comparison with the results from the current investigation shows a satisfactory agreement in the range of initial masses covered by the observations.

\subsection{Common findings and differences in AGB modelling}

To assess how the results presented here depend on the numerical details with which the AGB phase is modelled, in Fig. 7, 8 and 9 we compare the present findings with those published in Karakas (2014).

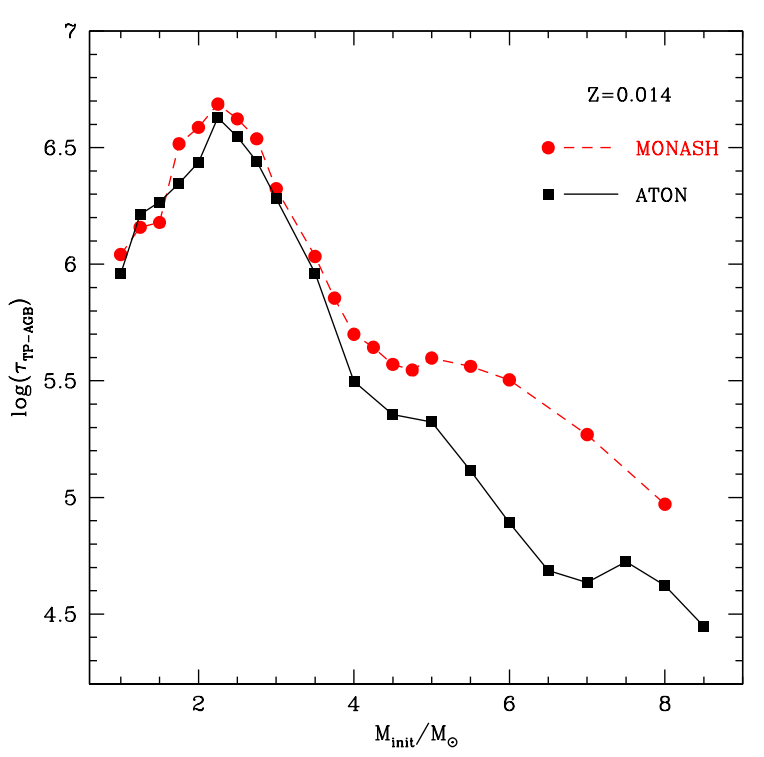

Figure 8. The duration of the TP-AGB phase of the AGB models presented here. The meaning of the symbols is the same as Fig. 7

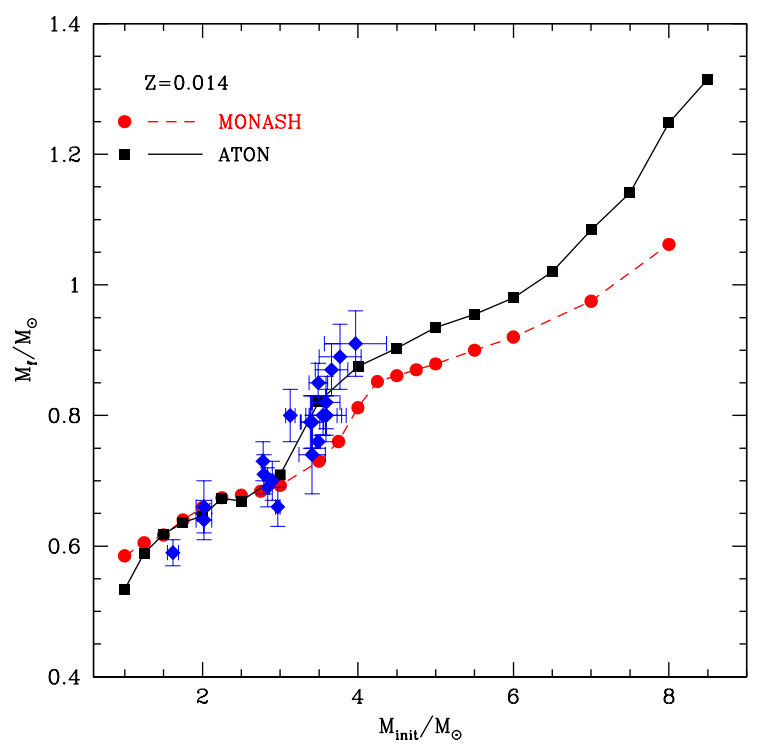

Figure 9. The initial - final mass relationship for the AGB models presented in Fig. 7 and 8 Blue diamonds indicate data from open clusters White Dwarfs by Kalirai et al. (2014).

In the large mass domain the luminosities reached by ATON models are generally higher than MONASH (see discussion in Section 4.1 and Fig. 2). The differences, as shown in Fig. 7 increase with the mass of the star. For an $8 M_{\odot}$ model the luminosity is $\sim 50 \%$ larger than in Karakas (2014). For stars of mass $3.5 M_{\odot} \leq M_{\text {init }} \leq 5 M_{\odot}$ the main actor is convection modelling, which affects the 
strength of the HBB experienced and thus the overall luminosity. Note that we do not need to consider the pre-AGB here because the core masses at the first TP are very similar (see Fig. 1). For higher mass stars the gap between the ATON and the MONASH luminosities is determined by the combined effects of convection modelling and the treatment of core overshoot during the MS phase. Overshoot during the main sequence means that the ATON models start the TP-AGB phase with larger core masses, which can be seen in Fig. 1 .

The differences in the luminosity reflect into the duration of the whole evolutionary phase. As shown in Fig. 8, the AGB evolutionary times of $M_{\text {init }} \geq 4 M_{\odot}$ stars are shorter in the ATON case. For the same reasons given above, the difference increases with the mass of the star, reaching a factor $\sim 3$ for the most massive AGB stars.

In the low mass domain the luminosities are very similar between the ATON and MONASH models because these stars do not experience HBB. The same holds for the duration of the entire AGB phase, reported in Fig. 8

For the initial-final mass relationship (see Fig. 9), we find once again similar results for models that do not have HBB. For the stars experiencing $\mathrm{HBB}$ we find that the ATON models develop more massive remnants compared to the MONASH case. As for the luminosity, we may associate these differences due the larger growth rate of the core mass of the ATON models (see the right panel of Fig. 2 and, for stars of mass $M_{\text {init }}>6 M_{\odot}$, to the difference in the core mass between ATON and MONASH models, present at the beginning of the TP-AGB phase.

\section{THE CHEMICAL COMPOSITION OF AGB STARS}

We focus now on the surface chemical composition, which is crucial to assess the role played by this class of objects in the pollution of the interstellar medium. Understanding how the surface chemistry changes as these stars evolve is also important to establish which kind of dust particles form in their wind (Ferrarotti \& Gail 2006). The latter will be discussed in more detail in Section 9, while some observational facts are discussed in Section 7 (with the exception of $\mathrm{Li}$, which is already discussed at the end of Section 6.3).

Fig. 10 shows the evolution of the surface abundances of carbon, nitrogen, oxygen, and sodium. For the CNO elements we refer to the most abundant isotopes, namely ${ }^{12} \mathrm{C}$, ${ }^{14} N$ and ${ }^{16} O$. The behaviour of carbon highlights the different evolution of $M_{\text {init }} \leq 3 M_{\odot}$ models from their highermass counterparts.

\subsection{Third dredge-up events: the formation of carbon stars}

Low-mass stars may undergo several TDU episodes, which increases the surface abundance of carbon. The maximum carbon abundance increases with increasing mass, up to $3 \mathrm{M}_{\odot}$. The overall increase in the surface carbon is a factor $\sim 2$, for $M=1.5 M_{\odot}$ models and up to a factor $\sim 4$, for models of $M=3 M_{\odot}$. The gas ejected by these stars is also enriched in nitrogen because of the first dredge-up (FDU); this can be seen in the steep rise of the surface nitrogen in the lines corresponding to $2 M_{\odot}$ and $3 M_{\odot}$ stars, in the right, top panel of Fig. 10

The enrichment in carbon favours the formation of carbon stars, when the surface $\mathrm{C} / \mathrm{O} \geq 1$. We find that at solar metallicities the minimum mass required to reach the carbon star stage is $1.5 M_{\odot}$. Stars below this limit loose their mantle before the $\mathrm{C} / \mathrm{O}>1$ condition is reached. For the majority of the AGB phase low-mass AGB stars are observed as oxygen-rich, as illustrated in Fig. 11. The duration of the C-rich phase is below $10 \%$ for the $2 M_{\odot}$ stars, whereas it is $\sim 15 \%$ for the $3 M_{\odot}$ star (see also Karakas \& Lattanzio 2014).

\subsection{The imprinting of $\mathrm{HBB}$ on the surface chemistry of massive AGB stars}

The HBB operating in massive AGB stars prevents the formation of a C-rich atmosphere and sets an upper limit for C-star formation. The upper mass limit is model dependent and is $3 M_{\odot}$ in the ATON models and $4.5 \mathrm{M}_{\odot}$ in the MONASH models.

The left, top panel of Fig. 10 shows that the mass expelled by these stars is carbon-poor, with a carbon content $\sim 20$ times smaller than the initial mass fraction. In the left, bottom panel of Fig. 10 we notice that the ejecta of massive AGB stars present traces of oxygen destruction: the most massive stars exhibit the largest depletion of oxygen, $\sim 30 \%$ lower than the initial abundance. The activation of the CNO cycles also results in a significant rise in the nitrogen abundance (see right, top panel of Fig. 10, which increases by a factor $\sim 20$ during the AGB evolution. The surface sodium abundance, shown in the right, bottom panel of Fig. 10, is seen to increase during the AGB phase, with production factors of the order of $\sim 4$. As discussed in section 4.1. the ejecta of these stars are sodium rich, owing to extremely favourable conditions to the synthesis of sodium.

While the gas expelled by massive AGB stars is expected to show the signature of proton-capture processing, the percentage of the AGB phase during which the surface chemical composition of the star is substantially altered by $\mathrm{HBB}$ is sensitive to the mass of the star. This can be deduced by focusing on the lines corresponding to the $4 M_{\odot}$ and $7 M_{\odot}$ stars in Fig. 10. In the former case the surface chemistry is practically unchanged for the first half of the evolution, whereas in the $7 M_{\odot}$ star, owing to an early activation of $\mathrm{HBB}$, the surface chemical composition show traces of HBB from the first TPs (see also Karakas \& Lugaro 2016). We conclude that in the massive AGB domain we shift gradually from the stars with mass just above the threshold to activate $\mathrm{HBB}$, which spend about half of their AGB evolution with the original chemical composition, to the most massive AGB stars, which show the imprinting of HBB for most of the TP-AGB phase.

\subsection{Lithium}

The discovery of bright red giants stars enriched in lithium in the Magellanic Clouds (Smith \& Lambert 1989, 1990) and our Milky Way Galaxy (García-Hernández et al. 2007. 2013 ) showed that AGB stars with HBB may be important factories for the production of $\mathrm{Li}$, at least for part of the AGB phase. 

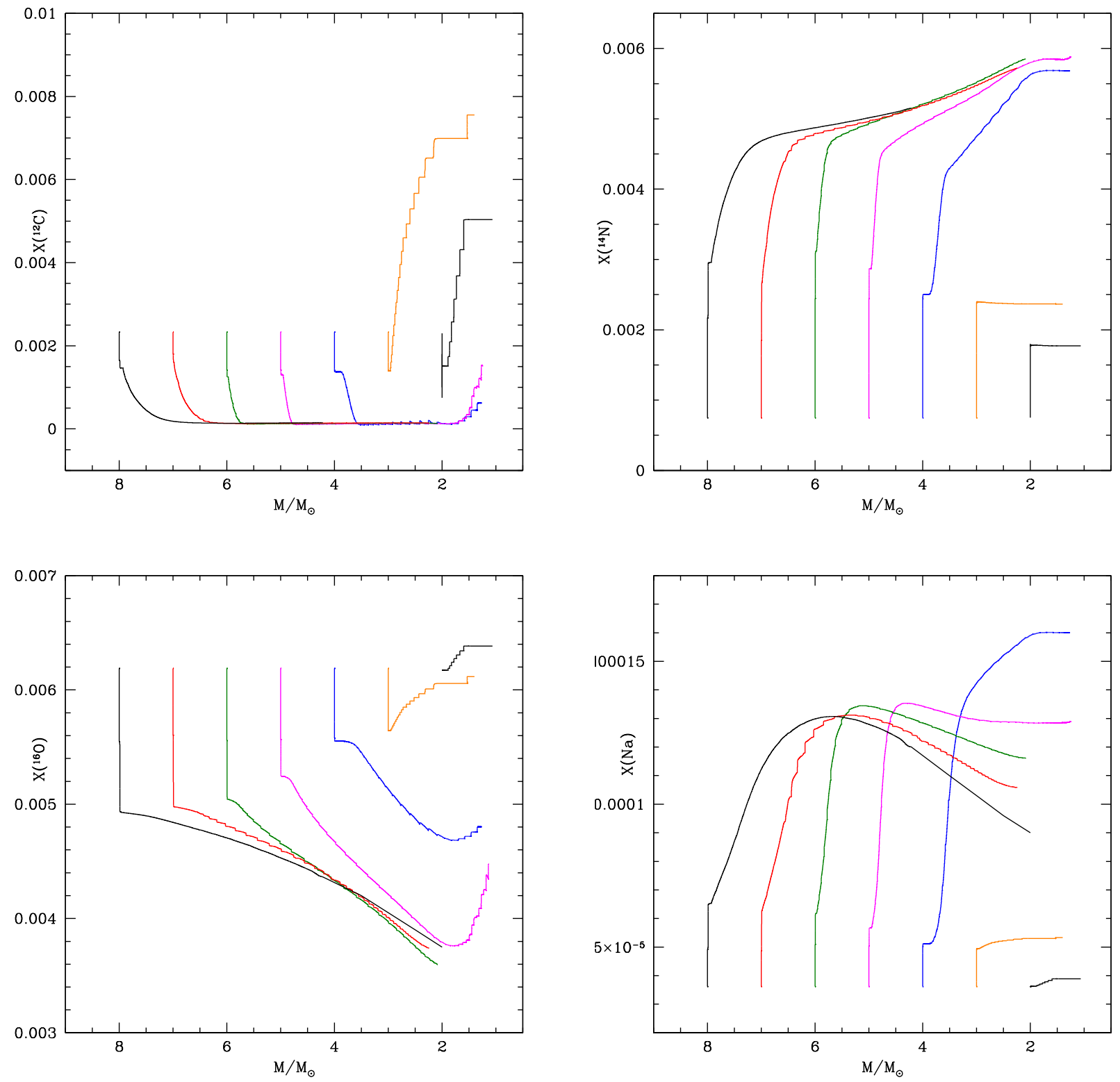

Figure 10. The variation of the surface mass fraction of the CNO elements and of sodium in AGB models of different initial mass. We report the current mass of the star on the abscissa to deduce the chemical composition of the ejecta. The starting point of each line marks the initial mass of the star.

The mechanism upon which lithium production is based was first identified by Cameron \& Fowler (1971) and confirmed by AGB modelling by Sackmann \& Boothroyd (1992). When the temperature at the base of the envelope, $T_{\text {bce }} \gtrsim 30 \mathrm{MK}$, the production of beryllium via the ${ }^{3} \mathrm{He}+{ }^{4} \mathrm{He} \rightarrow{ }^{7} \mathrm{Be}$ reaction is activated. Owing to the rapidity of convective motions, part of the beryllium is transported to cooler regions in the envelope where it can capture an electron to form lithium. The newly formed lithium will survive in the outer most layers although eventually convection will mix it down to hotter regions, where it will be destroyed. Lithium production will continue until the star runs out of ${ }^{3} \mathrm{He}$.

The temperatures given above require the ignition of HBB. Therefore lithium synthesis is limited to intermediatemass AGB stars, which is consistent with the existence of a lower limit in the luminosity of the lithium-rich sources in the Magellanic Clouds discovered by Smith \& Lambert (1989, 1990). The luminosity function of lithium-rich stars in the MCs was used by Ventura et al. (2000) to calibrate the rate of mass loss of oxygen-rich AGB stars.

Fig. 12 shows the variation of the surface lithium in models experiencing HBB. In the $y$-axis of the three panels 


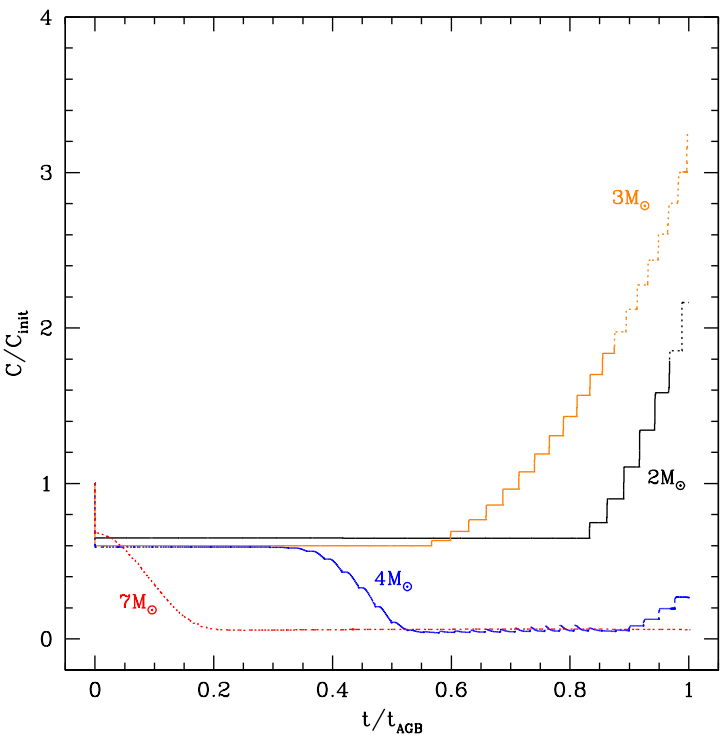

Figure 11. The variation of the surface carbon (normalized to the initial mass fraction) in AGB models of initial mass $2 M_{\odot}$ (black line), $3 M_{\odot}$ (orange), $4 M_{\odot}$ (blue), $7 M_{\odot}$ (red). Times on the abscissa are normalized to the duration of the AGB phase. The C-star phases are shown with a dotted line.

we show the standard quantity used to quantify the lithium content, namely $\log \left(\epsilon\left({ }^{7} L i\right)\right)=12+\log \left(n\left({ }^{7} L i\right) / n(H)\right)$. We note three points in common to all the stars considered: a) the stars enters the AGB phase with practically no lithium, as it is destroyed prior to the AGB; b) lithium production begins after the beginning of the AGB phase and the amount of lithium at the surface reaches a maximum abundance of the order of $\log \left(\epsilon\left({ }^{7} L i\right)\right) \sim 4.3$; c) the surface lithium declines in the final part of the AGB phase, when there is no ${ }^{3} \mathrm{He}$ available.

The left panel of Fig. 12 shows that the matter expelled by these stars is lithium-rich. The amount of $\mathrm{Li}$ enrichment increases with increasing stellar mass, because in massive AGB stars the rate at which mass loss occurs exceeds the rate in which ${ }^{3} \mathrm{He}$ is destroyed.

If we fix a threshold of $\log \left(\epsilon\left({ }^{7} L i\right)\right)=2$, above which we consider the star as being lithium-rich, we see in the middle panel of Fig. 12 that the lithium-rich phase is about half of the total $\mathrm{AGB}$ evolution. The most massive stars start to produce lithium during the very first interpulse phases, whereas in stars of lower mass the synthesis of lithium begins after $\sim 30 \%$ of the TP-AGB time has been completed, which is the time required to reach $\mathrm{HBB}$ conditions.

The right panel of Fig. 12 shows the lithium versus luminosity trend. A clear indication we get from this plot is that lithium-rich abundances are expected when the stars reach a luminosity of $20000 L_{\odot}$, i.e. $M_{b o l}=-6$, almost independently of the initial mass. This is the threshold above which we expect to find lithium-rich AGB stars. The upper limit in luminosity where we expect to observe lithium-rich sources is sensitive to the mass of the stars, and is higher in stars of higher initial mass.
The Lithium predictions, both for ATON and MONASH models (see e.g. García-Hernández et al. 2013), qualitatively agree with existing spectroscopic observations of massive Galactic HBB-AGB stars (García-Hernández et al. 2007, 2013), which show that the most luminous and Orich AGB stars (obscured OH/IR stars) in our Galaxy are Li-rich (García-Hernández et al. 2007) and that these stars can reach $\log (\epsilon(L i)) \sim 4$ at the beginning of the TP-AGB phase (García-Hernández et al. 2013). The s-process element $\mathrm{Rb}$, being a good indicator of the progenitor mass in AGB stars (see e.g. García-Hernández et al. 2006 Pérez-Mesa et al. 2017), has been also measured in these stars. Contrary to the synthesis of $\mathrm{Li}$, strong $\mathrm{Rb}$ production is expected towards the end of the AGB phase, when a significant number of TPs have been experienced (see e.g. García-Hernández et al. 2013). The observations show that the presence of $\mathrm{Li}$ is not always correlated with $\mathrm{Rb}$, indicating that the observed Galactic samples contain massive AGB stars with different progenitor masses and/or at several AGB evolutionary stages. A more detailed comparison with the observations is hampered by the uncertain distances (and so the their luminosities) to these Galactic massive AGB stars. Precise Gaia distances (and luminosities) to these Galactic massive AGB stars would permit to disentangle the evolutionary stage and progenitor mass of these Galactic Li-rich AGB stars.

\section{THE FINAL CHEMICAL COMPOSITION}

\subsection{Model predictions}

The final chemical composition is a key indicator of the relative efficiency of $\mathrm{HBB}$ and TDU in altering the surface chemistry of these stars.

Helium is a peculiar element among the various chemical species, because the surface abundance is not strongly sensitive to the details of AGB modelling. The modification of the surface helium content is mainly determined by the efficiency of the FDU and of the second dredge-up (SDU) episode.

Fig. 13 shows the final surface $\mathrm{He} / \mathrm{H}$ of the models discussed here. Stars of mass below $\sim 4 M_{\odot}$ do not experience any SDU. In this case the final $\mathrm{He} / \mathrm{H}$ ranges from $\sim 0.1$ to $\sim 0.11$, with little dependence on the mass of the star. In more massive stars the SDU, taking place shortly after the end of core He-burning (Karakas \& Lattanzio 2014), favours the increase in the surface helium. The strength of the SDU depends on the initial mass of the star and is more efficient for higher mass objects (Ventura 2010). As shown in Fig. 13 the final $\mathrm{He} / \mathrm{H}$ increases monotonically from $\mathrm{He} / \mathrm{H} \sim 0.11$, for a $4 M_{\odot}$ star, to $\mathrm{He} / H \sim 0.15$ for the most massive stars.

The comparison with the MONASH results is shown in Fig. 13 and outlines the following: a) in the low-mass domain the final $\mathrm{He} / \mathrm{H}$ is $\sim 0.05$ higher than the present models, owing to the higher helium assumed in the MONASH computations; b) for massive AGB stars we find a remarkable agreement between the ATON and the MONASH results. The helium enrichment of the surface regions of these stars turn out to be substantially independent of AGB modelling.

For the elements involved in CNO cycling Fig. 14 shows the final surface $\mathrm{C} / \mathrm{O}$ and $\mathrm{N} / \mathrm{O}$ ratios. These results can be easily interpreted based on the discussion in Section 6 . 

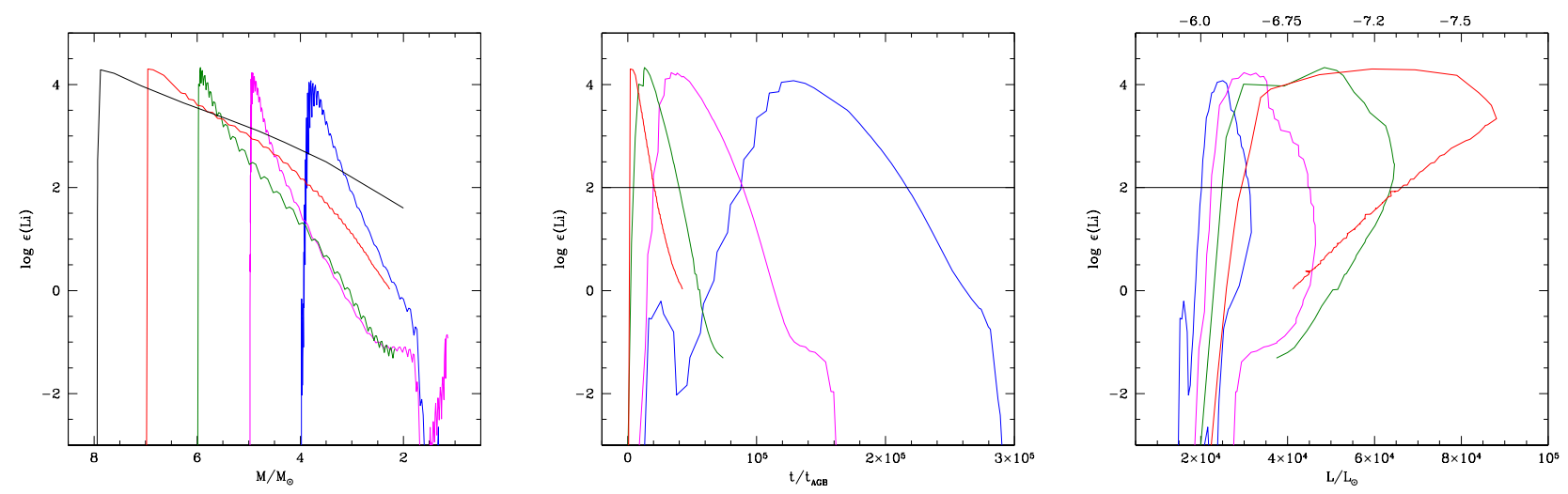

Figure 12. The variation of the surface lithium in AGB stars experiencing HBB. The various colours, the same used in Fig. 10 correspond to different initial masses. The surface lithium is shown as a function of the current mass of the star (left panel), of the time counted since the beginning of the AGB evolution, normalized to the total duration of the AGB phase (middle panel) and of the luminosity of the star. On top of the right panel we show the bolometric magnitudes corresponding to the luminosities reported on the abscissa. In the middle and right panels the track of the $8 M_{\odot}$ star was omitted for clarity reasons, as it would largely overlay with the $7 M_{\odot}$ line.

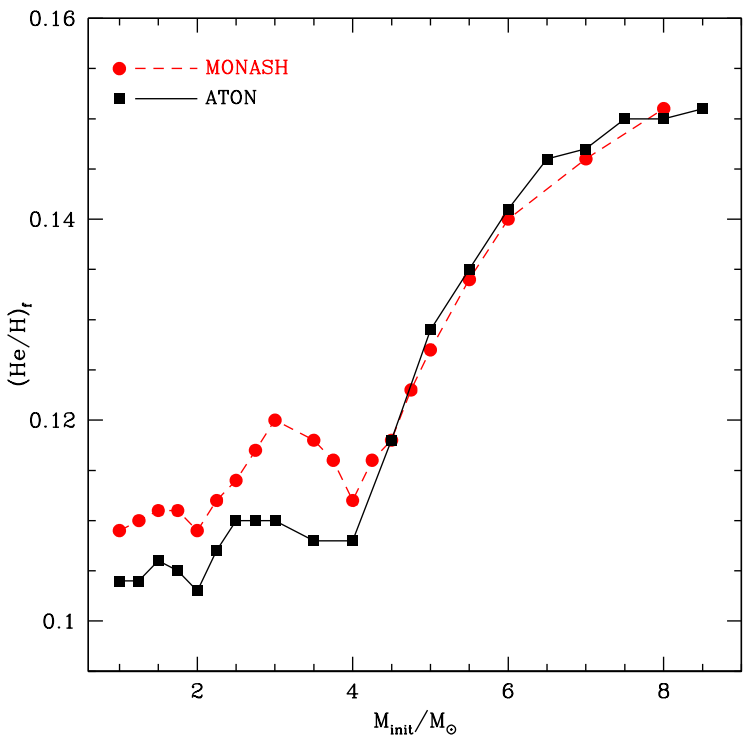

Figure 13. The final $\mathrm{He} / \mathrm{H}$ fraction of AGB models of different mass.

For stars of mass below $3.5 M_{\odot}$ the final C/O increases with the mass of the star, ranging from 0.4 ( $1 M_{\odot}$ star) to $2.5\left(3 M_{\odot}\right)$. This is because the number of TDU episodes increases with stellar mass, which in turn increases the final $\mathrm{C} / \mathrm{O}$ ratio. We have seen that stars in the mass range $1.5 M_{\odot} \leq M_{\text {init }} \leq 3 M_{\odot}$ become carbon stars; this is consistent with their final $\mathrm{C} / \mathrm{O}$ ratios above unity. The final $\mathrm{N} / \mathrm{O}$ shows up only a mild dependance on the stellar mass, which is caused by the efficiency of the FDU (e.g., Boothroyd \& Sachmann 1999). The final N/O for these stars spans the range $0.2<N / O<0.4$.

The stars experiencing HBB follow a completely dif- ferent behaviour. As shown in the left panel of Fig. 14 we find $\mathrm{C} / \mathrm{O}$ ratios below 0.1 , independently of the stellar mass. These stars also show a significant increase in the final $\mathrm{N} / \mathrm{O}$, with values in the range $1.5<N / O<2$.

The comparison with the results from Karakas (2014) outlines strong similarities in the low-mass domain, whereas the ATON findings for massive AGB stars reveal significant differences compared to MONASH models.

First, we find that in the range of mass $3 M_{\odot} \leq M_{\text {init }} \leq$ $4 M_{\odot}$ the MONASH $\mathrm{C} / \mathrm{O}$ ratios are $\lesssim 2$, whereas the corresponding ATON values are $\mathrm{C} / \mathrm{O}<0.2$. This is due to a shift in the threshold mass required to ignite $\mathrm{HBB}$, which is $\sim 1 M_{\odot}$ higher in the MONASH models.

For stars of mass above $4 M_{\odot}$, while the present models are characterized by final $\mathrm{C} / \mathrm{O}$ ratios below 0.1 , in the MONASH models we find $0.5<C / O<1.5$ (see Fig 2 in Karakas \& Lugaro 2016). This is partly due to the stronger HBB found in the present models, owing to the use of the FST model for convection. An additional explanation is that the TDU efficiency is extremely poor in this mass domain, whereas in the MONASH models some carbon is transported to the surface via TDU, despite the fact that some of the carbon is subsequently destroyed by HBB during the following interpulse phase. This explanation finds additional confirmation in the comparison of the final N/O ratios, which are higher in the MONASH models. This is because of the additional contribution of primary nitrogen, which is synthesized by fresh carbon dredged-up from the He-shell.

\subsection{Observational facts and future directions}

A detailed comparison with the composition of solar metallicity AGB, post-AGB stars and PNe, although out of the scope of the present paper, would offer, in principle, the opportunity to test the theoretical models of this still rather uncertain evolutionary phase. Ideally, the predicted abundances of $\mathrm{He}, \mathrm{C}, \mathrm{N}, \mathrm{O}, \mathrm{Ne}, \mathrm{Na}, \mathrm{Mg}$, and $\mathrm{Al}$ (as well as some key abundance ratios like $\mathrm{C} / \mathrm{O}, \mathrm{N} / \mathrm{O}$, and $\mathrm{C} / \mathrm{N}$ ), from 

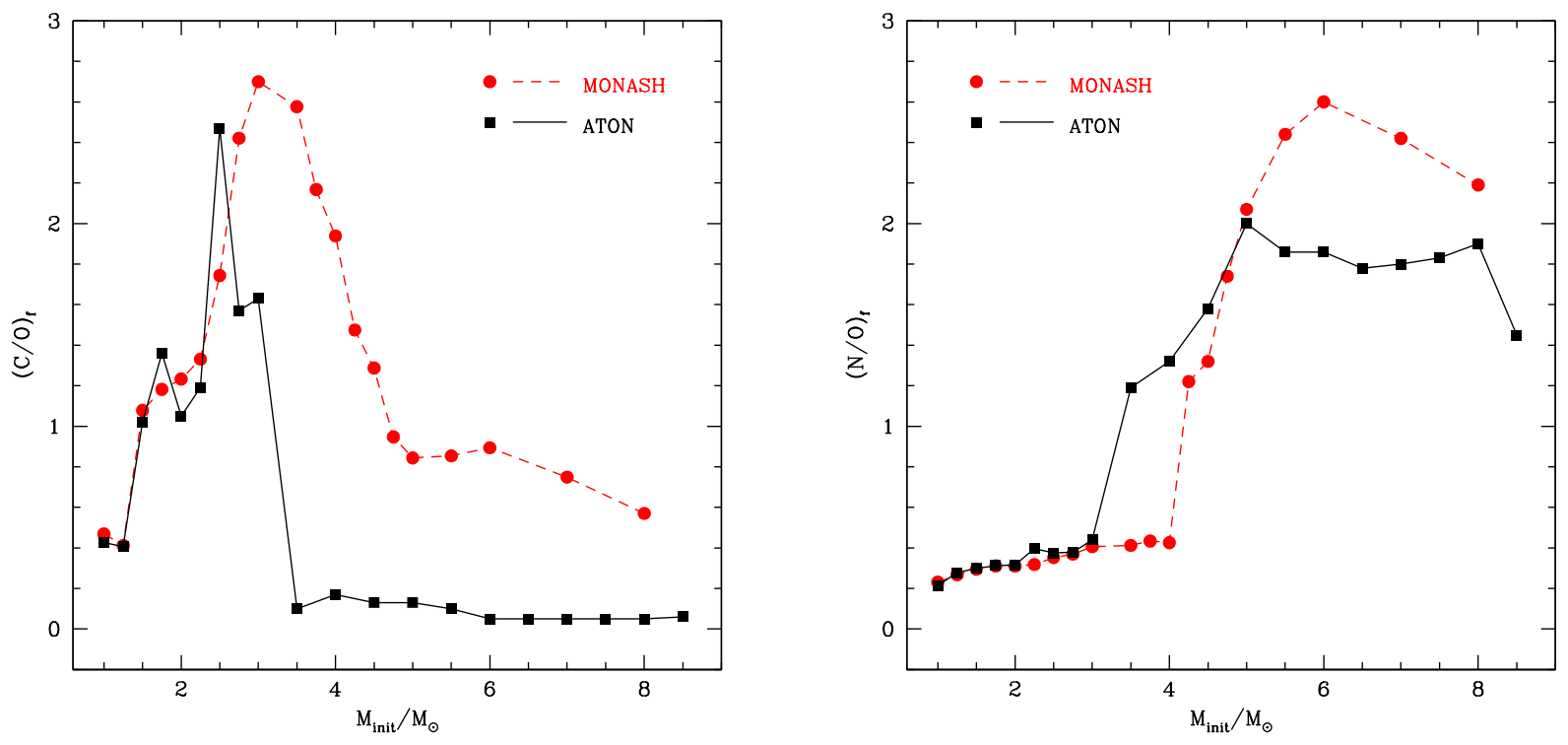

Figure 14. The final $\mathrm{C} / \mathrm{O}$ (left panel) and N/O (right) ratios for AGB stars of different mass

the two AGB models (ATON vs. MONASH) discussed here, could be compared with the abundances of these elements as observed in solar metallicitiy Galactic AGB, post-AGB stars and $\mathrm{PNe}$ and available in the literature. However, this is not an easy task and there are several observational limitations that depend on the source type and enumerated below. Another serious observational problem is that Galactic AGB and post-AGB stars, and PNe are plagued by distance uncertainties, which avoid detailed studies of AGB nucleosynthesis at solar metallicity, depending on progenitor mass and luminosity; the Gaia mission is thus expected to overcome the latter severe observational problem.

i) AGB stars: both C-rich and O-rich AGB stars may not display the final chemical composition and their chemical abundance analysis (especially towards the end of the AGB, where they are usually dust enshrouded; i.e., optically invisible) is very complicated due to their complex dynamical atmospheres, which can dramatically affect the derived abundances (see e.g. Zamora et al. 2014 Pérez-Mesa et al. 2017). The CNO elemental and isotopic abundances, as obtained from high-resolution optical and/or near-IR spectroscopy, are only available in some Galactic C-rich AGB stars (e.g. Hedrosa et al. 2013 Abia et al. 2017, and references therein). In the more massive O-rich HBB AGB stars the $\mathrm{CNO}$ elemental/isotopic ratios can be derived in the near-IR wavelength region only and such near-IR measurements have not been reported yet. Other elements such as He and Ne cannot be measured in AGB stars, while to the best of our knowledge, the abundances of $\mathrm{Na}, \mathrm{Mg}$, and $\mathrm{Al}$ (although measurable from near-IR spectra) in Galactic AGB stars have still to be reported. On-going massive highresolution near-IR spectroscopic surveys such as the second generation of The Apache Point Observatory Galactic Evolution Experiment (APOGEE-2; see e.g. Blanton et al.|2017) are expected to represent a major step forward in our un- derstanding of AGB nucleosynthesis, offering a invaluable test of the theoretical models presented here. APOGEE2 will provide homogeneous $\mathrm{CNO}$ elemental and isotopic abundances (at least for the ${ }^{12} \mathrm{C} /{ }^{13} \mathrm{C}$ ratio ${ }^{3}$ ) as well as $\mathrm{Na}$, $\mathrm{Mg}$, and $\mathrm{Al}$ abundances for complete (flux-limited) samples of Galactic AGB stars (bulge, disk, and halo), covering all progenitor masses. The possible circumstellar effects (if any) on the near-IR molecular $(\mathrm{CO}, \mathrm{OH}, \mathrm{CN})$ and atomic lines $(\mathrm{Al}, \mathrm{Mg}, \mathrm{Na})$ remained to be explored. Finally, observations of heavy neutron-rich elements in AGB stars may provide clues to test these theoretical models but their uncertainties are very large, ranging from $0.3-0.4$ dex to as high as 0.7 dex for Rb (e.g., Abia et al. 2001 García-Hernández et al. 2006 Pérez-Mesa et al. 2017), highlighting the need for independent complementary observations (e.g., in post-AGB stars and PNe; see below). Also, the simulations of the nucleosynthesis due to slow-neutron captures (the s-process) in the ATON AGB models are still under construction (Yagüe et al. 2016).

ii) Post-AGB stars: The atmospheres of these stars (stars in the fast transition phase between AGB stars and PNe; see e.g., Van Winckel 2003, for a review) display the final chemical composition (i.e., the final result of chemical enrichment from internal nucleosynthesis and dredgeup processes during the entire stellar evolution), being, in principle, ideal probes to study AGB stellar nucleosynthe-

3 Di Criscienzo et al. (2016) has recently compared the observed $\mathrm{C}$ and $\mathrm{O}$ isotopic ratios (i.e., ${ }^{12} \mathrm{C} /{ }^{13} \mathrm{C}$ and ${ }^{16} \mathrm{O} /{ }^{17} \mathrm{O} /{ }^{18} \mathrm{O}$ ) available in the literature for several types of AGB stars with the AGB ATON predictions. The available $\mathrm{C}$ and $\mathrm{O}$ isotopic ratios, however, are not homogeneous and they come from different observational data; from optical/near-IR spectra in C-rich AGB stars to the far-IR (in a few massive HBB O-rich stars) and to the radio domain. 
sis. Their photospheres (spectral types from K to A; see e.g., Suárez et al. 2006) are hotter than those in AGB stars, dominated by atomic spectral lines that allow for more accurate abundance determinations of a larger number of elements, including $\mathrm{C}, \mathrm{N}, \mathrm{O}, \mathrm{Na}, \mathrm{Mg}$, and $\mathrm{Al}$, among others, but also many neutron-rich s-process elements (see e.g. De Smedt et al. 2016) (as mentioned above, the s-process ATON simulations are still under construction). The use of the chemical composition observed in post-AGB stars as valuable tests for the theoretical AGB models, however, is also hampered by the non-homogeneous chemical analysis reported in the literature and because the number of elements that can be measured in post-AGB stars depend on the stellar effective temperatures. Because of the fast AGB-PNe transition times $\left(\sim 10^{2}-10^{4}\right.$ years, depending on the initial mass; see e.g. Vassiliadis \& Wood 1994 only about hundred confirmed post-AGB stars are known in the Galaxy (e.g., Szczerba et al. 2007, 2012). In addition, only a handful of post-AGB stars have been observed at high-resolution in the optical range. Finally, present spectroscopic optical observations of post-AGB stars are strongly biased towards the lower mass progenitors (say $\sim 1-2 \mathrm{M}_{\odot}$ ); e.g., usually high Galactic latitude (i.e., metal-poor) and optically bright s-process enriched C-rich post-AGB stars (see e.g. Van Winckel \& Reyniers 2000: Reyniers et al. 2007; De Smedt et al.|2016). In contrast, higher mass post-AGB stars (above $2 \mathrm{M}_{\odot}$ ), evolving much faster, may have systematically escaped detection in past high-resolution optical surveys because they may remain hidden (dust enshrouded) during the whole AGBPN transition. Both optically bright and obscured post-AGB stars could be studied in the near-IR, which provides an unexplored spectral window that should be exploited in order to get homogeneous chemical analysis of a complete sample of Galactic post-AGB stars. As in the case of Galactic AGB stars, the SDSS-IV/APOGEE-2 survey could provide such an ambitious goal; e.g., SDSS-IV/APOGEE-2 may discover the coolest post-AGB stars ( $\mathrm{K}$ and $\mathrm{M}$ spectral types) in our Galaxy with access to the dust enshrouded ones (in principle the more massive ones), no accesible in the optical.

iii) PNe: The comparison of the theoretical predictions with the chemical composition observed in PNe (via their nebular emission lines) offers another opportunity to test theoretical models of the still rather uncertain AGB phase.

A recent step in this direction was done by Ventura et al. (2017), who used the observed nebular chemical composition to estimate the mass and formation epoch of the progenitors of 142 Galactic PNe. This analysis was based on the comparison of the abundances data with the ATON AGB model predictions presented here, specifically on the final abundances of the various chemical species, discussed in this section. On general grounds, the chemical abundances in PNe, typically more accurate than those in AGB stars, have also their own problems/limitations; e.g., the chemical abundances available in the literature, again, are not completely homogeneous and ionization correction factors (ICFs), sometimes very uncertain, are needed to estimate the contribution of unobserved ions to the total abundances (see e.g., Delgado-Inglada, Morisset \& Stasińska 2014, and references therein). However, the main advantages of $\mathrm{PNe}$ (with respect to AGB and post-AGB stars, see above) are that PNe can be easily observed at very large distances (because of their emission-line nature) and that known $\mathrm{PNe}$ samples are more complete (e.g., they cover the full range of initial masses, despite the masses estimated are more uncertain compared to post-AGB stars). Also, the abundances of key elements such as $\mathrm{He}, \mathrm{C}, \mathrm{N}, \mathrm{O}$, and $\mathrm{Ne}$ are accesible for all types of $\mathrm{PNe}$; recent studies outlined the possibility of measuring the surface $\mathrm{Zn}$ (Smith et al. 2017). On the other hand, the abundances of $\mathrm{Na}, \mathrm{Mg}$, and $\mathrm{Al}$ cannot be measured in PNe. The abundances of He, N, O, and Ne (among others like $\mathrm{Ar}, \mathrm{Cl}$, and $\mathrm{S}$ ) are easily extracted from low-resolution optical spectroscopy (see e.g., García-Hernández \& Górny 2014 and references therein) and available in the literature. However, the derivation of $\mathrm{C}$ abundances needs deep highresolution optical spectro ${ }^{4}$ and/or UV spectra (e.g., by using the Hubble Space Telescope, HST), which are not easily obtained (see e.g., Ventura et al.|2017). For example, the availability of accurate $\mathrm{C}$ abundances from HST-UV spectra in $\mathrm{PNe}$ of the Magellanic Clouds, together with other observational data such as optical and mid-IR spectra, have permitted detailed comparisons of their CNO elemental abundances with the predictions from the ATON AGB models (Ventura et al. 2015b, 2016c). Similar studies in complete samples of Galactic PNe are not still possible, mainly due to the lack of UV spectra available for only a few Galactic sources (see e.g., Ventura et al. 2017). Thus, the collection of deep high-resolution optical/near-IR spectra and/or UV spectra in a complete sample of Galactic PNe would permit to construct a unique homogeneous database of $\mathrm{PNe}$ nebular abundances to test the AGB theoretical models. Unfortunately, deep high-resolution optical/near-IR nebular spectroscopy is very time consuming (even with 8-10 m class telescopes), while UV spectroscopy requires the use of precious HST time.

\section{YIELDS FROM AGB STARS}

The yields of the various chemical species are key quantities to understand the pollution expected from a class of stars and the way they participate in the gas cycle of the interstellar medium.

In the following we will use the classic definition, according to which we indicate the yield $Y_{i}$ of the $i$-th element as

$$
Y_{i}=\int\left[X_{i}-X_{i}^{i n i t}\right] \dot{M} d t .
$$

The integral is calculated over entire stellar lifetime and $X_{i}^{i n i t}$ is the mass fraction of species $i$ at the beginning of the evolution. Based on this definition, the yield is negative if an element is destroyed and positive if it is produced over the life of the star.

Fig. 15 shows the yields of helium, $Y_{H e}$. It is evident the sudden increase in $Y_{H e}$ occurring at $\sim 4 M_{\odot}$, representing the lower limit for solar metallicity stars to experience SDU. The trend of $Y_{H e}$ with the mass of the star is positive, ranging from $Y_{H e}=0.1 M_{\odot}$ to $Y_{H e}=0.75 M_{\odot}$. This

\footnotetext{
4 Some heavy s-process elements like Se, $\mathrm{Kr}, \mathrm{Xe}, \mathrm{Rb}, \mathrm{Cd}$, and Ge can be also obtained from deep high-resolution optical and/or near-IR spectroscopy (e.g., Sharpee et al. 2007, Sterling \& Dinerstein 2008, Sterling et al. 2016).
} 
Table 2. Chemical yields (see text for definition) for solar metallicity, AGB models

\begin{tabular}{|c|c|c|c|c|c|c|c|c|c|c|c|c|c|c|}
\hline$M$ & $H$ & $\mathrm{He}$ & ${ }^{12} C$ & ${ }^{13} \mathrm{C}$ & ${ }^{14} N$ & ${ }^{16} \mathrm{O}$ & ${ }^{17} \mathrm{O}$ & ${ }^{18} \mathrm{O}$ & $\mathrm{Ne}$ & ${ }^{23} \mathrm{Na}$ & ${ }^{24} M g$ & ${ }^{25} \mathrm{Mg}$ & ${ }^{26} \mathrm{Mg}$ & ${ }^{27} \mathrm{Al}$ \\
\hline 1.00 & $-1.3 \mathrm{E}-2$ & $1.3 \mathrm{E}-2$ & $-1.8 \mathrm{E}-4$ & $-5.6 \mathrm{E}-6$ & $2.1 \mathrm{E}-4$ & - & - & - & - & - & - & - & - & - \\
\hline 1.25 & $-1.7 \mathrm{E}-2$ & $1.4 \mathrm{E}-2$ & $1.1 \mathrm{E}-3$ & $2.6 \mathrm{E}-5$ & $3.2 \mathrm{E}-4$ & $7.4 \mathrm{E}-5$ & - & - & $4.5 \mathrm{E}-5$ & $7.9 \mathrm{E}-7$ & - & $3.4 \mathrm{E}-7$ & $3.4 \mathrm{E}-7$ & - \\
\hline 1.50 & $-1.9 \mathrm{E}-2$ & $1.8 \mathrm{E}-2$ & $2.5 \mathrm{E}-4$ & $-4.2 \mathrm{E}-5$ & $7.0 \mathrm{E}-4$ & $1.1 \mathrm{E}-4$ & $2.7 \mathrm{E}-6$ & $-2.1 \mathrm{E}-6$ & $3.2 \mathrm{E}-5$ & $7.1 \mathrm{E}-7$ & $-1.2 \mathrm{E}-8$ & $2.2 \mathrm{E}-7$ & $4.0 \mathrm{E}-7$ & $2.5 \mathrm{E}-7$ \\
\hline 1.75 & $-2.3 \mathrm{E}-2$ & $2.0 \mathrm{E}-2$ & $1.9 \mathrm{E}-3$ & $-7.1 \mathrm{E}-5$ & $1.1 \mathrm{E}-3$ & $1.5 \mathrm{E}-4$ & $1.2 \mathrm{E}-5$ & $-3.5 \mathrm{E}-6$ & $1.3 \mathrm{E}-4$ & $2.2 \mathrm{E}-6$ & $-2.3 \mathrm{E}-7$ & $2.1 \mathrm{E}-6$ & $2.1 \mathrm{E}-6$ & $9.5 \mathrm{E}-7$ \\
\hline 2.00 & $-3.0 \mathrm{E}-2$ & $2.6 \mathrm{E}-2$ & $2.7 \mathrm{E}-3$ & $-9.2 \mathrm{E}-5$ & $1.4 \mathrm{E}-3$ & $2.1 \mathrm{E}-4$ & $1.6 \mathrm{E}-5$ & $-4.6 \mathrm{E}-6$ & $1.8 \mathrm{E}-4$ & $2.9 \mathrm{E}-6$ & $-3.9 \mathrm{E}-7$ & $3.3 \mathrm{E}-6$ & $3.1 \mathrm{E}-6$ & $1.4 \mathrm{E}-6$ \\
\hline 2.25 & $-4.5 \mathrm{E}-2$ & $4.0 \mathrm{E}-2$ & $3.6 \mathrm{E}-3$ & $-1.2 \mathrm{E}-4$ & $2.2 \mathrm{E}-3$ & $1.8 \mathrm{E}-4$ & $2.4 \mathrm{E}-5$ & $-6.0 \mathrm{E}-6$ & $2.3 \mathrm{E}-4$ & $1.9 \mathrm{E}-5$ & $-8.4 \mathrm{E}-7$ & $6.3 \mathrm{E}-6$ & $6.3 \mathrm{E}-6$ & $2.5 \mathrm{E}-6$ \\
\hline 2.50 & $-6.7 \mathrm{E}-2$ & $5.4 \mathrm{E}-2$ & $9.7 \mathrm{E}-3$ & $-1.5 \mathrm{E}-4$ & $2.7 \mathrm{E}-3$ & $1.1 \mathrm{E}-4$ & $2.2 \mathrm{E}-5$ & $-7.3 \mathrm{E}-6$ & $5.4 \mathrm{E}-4$ & $2.8 \mathrm{E}-5$ & $-4.2 \mathrm{E}-6$ & $3.1 \mathrm{E}-5$ & $2.0 \mathrm{E}-5$ & $9.9 \mathrm{E}-6$ \\
\hline 2.75 & $-7.4 \mathrm{E}-2$ & $6.4 \mathrm{E}-2$ & $7.4 \mathrm{E}-3$ & $-1.6 \mathrm{E}-4$ & $3.2 \mathrm{E}-3$ & $-3.6 \mathrm{E}-4$ & $2.1 \mathrm{E}-5$ & $-8.2 \mathrm{E}-6$ & $4.7 \mathrm{E}-4$ & $3.2 \mathrm{E}-5$ & $-3.9 \mathrm{E}-6$ & $2.5 \mathrm{E}-5$ & $1.8 \mathrm{E}-5$ & $1.1 \mathrm{E}-5$ \\
\hline 3.00 & $-8.7 \mathrm{E}-2$ & $7.4 \mathrm{E}-2$ & $9.3 \mathrm{E}-3$ & $-1.9 \mathrm{E}-4$ & $3.7 \mathrm{E}-3$ & $-4.1 \mathrm{E}-4$ & $2.1 \mathrm{E}-5$ & $-9.4 \mathrm{E}-6$ & $6.0 \mathrm{E}-4$ & $3.8 \mathrm{E}-5$ & $-6.3 \mathrm{E}-6$ & $3.8 \mathrm{E}-5$ & $2.6 \mathrm{E}-5$ & $1.6 \mathrm{E}-5$ \\
\hline 3.50 & $-8.2 \mathrm{E}-2$ & 7.7E-2 & $-3.7 \mathrm{E}-3$ & $-2.7 \mathrm{E}-4$ & $1.0 \mathrm{E}-2$ & $-1.3 \mathrm{E}-3$ & $1.8 \mathrm{E}-5$ & $-2.9 \mathrm{E}-5$ & $8.2 \mathrm{E}-5$ & $1.1 \mathrm{E}-4$ & $-6.3 \mathrm{E}-6$ & $9.6 \mathrm{E}-6$ & $1.5 \mathrm{E}-5$ & $1.5 \mathrm{E}-5$ \\
\hline 4.00 & $-9.0 \mathrm{E}-2$ & $8.8 \mathrm{E}-2$ & $-6.3 \mathrm{E}-3$ & $-7.3 \mathrm{E}-4$ & $1.3 \mathrm{E}-2$ & $-3.7 \mathrm{E}-3$ & $2.9 \mathrm{E}-5$ & $-4.0 \mathrm{E}-5$ & $-2.3 \mathrm{E}-4$ & $3.2 \mathrm{E}-4$ & $-2.0 \mathrm{E}-5$ & $8.9 \mathrm{E}-6$ & $1.6 \mathrm{E}-5$ & $9.9 \mathrm{E}-6$ \\
\hline 4.50 & $-1.7 \mathrm{E}-1$ & $1.7 \mathrm{E}-1$ & $-7.6 \mathrm{E}-3$ & $-8.8 \mathrm{E}-4$ & $1.6 \mathrm{E}-2$ & $-6.0 \mathrm{E}-3$ & $3.6 \mathrm{E}-5$ & $-4.7 \mathrm{E}-5$ & $-3.0 \mathrm{E}-4$ & $3.4 \mathrm{E}-4$ & $-6.8 \mathrm{E}-5$ & $3.7 \mathrm{E}-5$ & $2.2 \mathrm{E}-5$ & $9.2 \mathrm{E}-6$ \\
\hline 5.00 & $-2.6 \mathrm{E}-1$ & $2.6 \mathrm{E}-1$ & $-8.3 \mathrm{E}-3$ & $-9.5 \mathrm{E}-4$ & $1.9 \mathrm{E}-2$ & $-7.5 \mathrm{E}-3$ & $4.5 \mathrm{E}-5$ & $-5.3 \mathrm{E}-5$ & $-2.8 \mathrm{E}-4$ & $3.7 \mathrm{E}-4$ & $-1.5 \mathrm{E}-4$ & $1.2 \mathrm{E}-4$ & $2.0 \mathrm{E}-5$ & $5.2 \mathrm{E}-5$ \\
\hline 5.50 & $-3.5 \mathrm{E}-1$ & $3.5 \mathrm{E}-1$ & $-9.8 \mathrm{E}-3$ & $-1.1 \mathrm{E}-3$ & $2.1 \mathrm{E}-2$ & $-9.2 \mathrm{E}-3$ & $5.5 \mathrm{E}-5$ & $-6.0 \mathrm{E}-5$ & $-3.8 \mathrm{E}-4$ & $4.0 \mathrm{E}-4$ & $-2.5 \mathrm{E}-4$ & $1.9 \mathrm{E}-4$ & $4.1 \mathrm{E}-5$ & $1.3 \mathrm{E}-5$ \\
\hline 6.00 & $-4.4 \mathrm{E}-1$ & $4.4 \mathrm{E}-1$ & $-1.1 \mathrm{E}-2$ & $-1.2 \mathrm{E}-3$ & $2.3 \mathrm{E}-2$ & $-1.0 \mathrm{E}-2$ & $7.0 \mathrm{E}-5$ & $-6.6 \mathrm{E}-5$ & $-4.1 \mathrm{E}-4$ & $4.3 \mathrm{E}-4$ & $-3.8 \mathrm{E}-4$ & $3.1 \mathrm{E}-4$ & $5.1 \mathrm{E}-5$ & $1.3 \mathrm{E}-5$ \\
\hline 6.50 & $-5.2 \mathrm{E}-1$ & $5.2 \mathrm{E}-1$ & $-1.2 \mathrm{E}-2$ & $-1.4 \mathrm{E}-3$ & $2.5 \mathrm{E}-2$ & $-1.1 \mathrm{E}-2$ & $9.0 \mathrm{E}-5$ & $-7.2 \mathrm{E}-5$ & $-4.4 \mathrm{E}-4$ & 4.6E-4 & $-5.3 \mathrm{E}-4$ & $4.6 \mathrm{E}-4$ & $3.7 \mathrm{E}-5$ & $5.6 \mathrm{E}-5$ \\
\hline 7.00 & $-5.7 \mathrm{E}-1$ & $5.7 \mathrm{E}-1$ & $-1.3 \mathrm{E}-2$ & $-1.4 \mathrm{E}-3$ & $2.6 \mathrm{E}-2$ & $-1.1 \mathrm{E}-2$ & $1.2 \mathrm{E}-4$ & $-7.8 \mathrm{E}-5$ & $-4.6 \mathrm{E}-4$ & $4.8 \mathrm{E}-4$ & $-6.9 \mathrm{E}-4$ & $6.2 \mathrm{E}-4$ & $4.0 \mathrm{E}-5$ & $5.3 \mathrm{E}-5$ \\
\hline 7.50 & $-6.5 \mathrm{E}-1$ & $6.5 \mathrm{E}-1$ & $-1.4 \mathrm{E}-2$ & $-1.5 \mathrm{E}-3$ & $2.9 \mathrm{E}-2$ & $-1.3 \mathrm{E}-2$ & $1.2 \mathrm{E}-4$ & $-8.2 \mathrm{E}-5$ & $-5.0 \mathrm{E}-4$ & $5.2 \mathrm{E}-4$ & $-7.4 \mathrm{E}-4$ & $6.8 \mathrm{E}-4$ & $3.9 \mathrm{E}-5$ & $6.8 \mathrm{E}-5$ \\
\hline 8.00 & $-6.5 \mathrm{E}-1$ & $6.5 \mathrm{E}-1$ & $-1.4 \mathrm{E}-2$ & $-1.6 \mathrm{E}-3$ & $2.9 \mathrm{E}-2$ & $-1.2 \mathrm{E}-2$ & $1.6 \mathrm{E}-4$ & $-8.8 \mathrm{E}-5$ & $-5.2 \mathrm{E}-4$ & $5.2 \mathrm{E}-4$ & $-8.7 \mathrm{E}-4$ & $8.1 \mathrm{E}-4$ & $3.7 \mathrm{E}-5$ & $6.8 \mathrm{E}-5$ \\
\hline 8.50 & $-7.5 \mathrm{E}-1$ & $7.5 \mathrm{E}-1$ & $-1.5 \mathrm{E}-2$ & $-1.5 \mathrm{E}-3$ & $3.0 \mathrm{E}-2$ & $-1.2 \mathrm{E}-2$ & $1.6 \mathrm{E}-4$ & $-9.3 \mathrm{E}-5$ & $-3.4 \mathrm{E}-4$ & $6.2 \mathrm{E}-4$ & $-1.2 \mathrm{E}-3$ & $1.1 \mathrm{E}-3$ & $3.6 \mathrm{E}-5$ & $7.5 \mathrm{E}-5$ \\
\hline
\end{tabular}

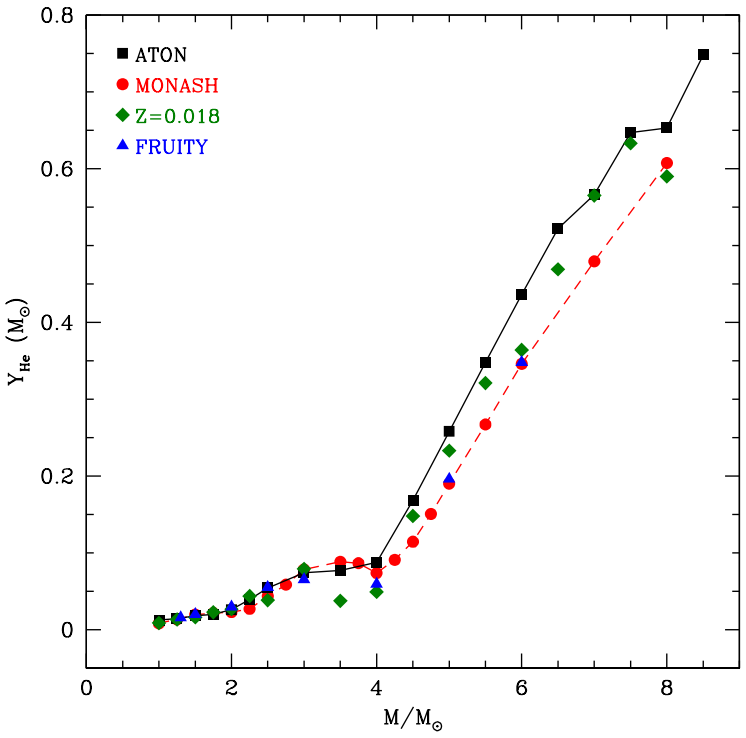

Figure 15. The helium yields (see text for definition) of solar metallicity stars of different mass are indicated with black squares. The results by Karakas \& Lugaro (2016) (red points), Cristallo et al. (2015) (blue triangles) and Di Criscienzo et al. (2016) (green diamonds) are also shown for comparison.

is in agreement with the results shown in Fig. 13 In the low-mass domain the helium yield is determined primarily by FDU and we find $Y_{H e}<0.1 M_{\odot}$ in all cases.

The yields of the main CNO species and of sodium are shown in Fig. 16. The carbon yields can be understood based on the different behaviour of low-mass stars and massive AGB stars, outlined in the previous sessions. We find car- bon production for stars of mass $M_{\text {init }} \leq 3 M_{\odot}$, with a maximum yield of $\sim 0.01 M_{\odot}$ for $2.5-3 M_{\odot}$ stars, which experience the largest enrichment of carbon at the surface (see Fig. 10). $Y_{\mathrm{C}}$ increases with stellar mass, in agreement with the discussion in section 6 For stars experiencing HBB the carbon yields are negative. In this mass domain $Y_{\mathrm{C}}$ decreases from $Y_{\mathrm{C}}=-0.005 M_{\odot}$ (for the $3.5 M_{\odot}$ star) to $Y_{\mathrm{C}}=-0.015 M_{\odot}\left(3.5 M_{\odot}\right)$.

The oxygen yields of low-mass stars are almost zero because little oxygen is produced in the He-intershell (see Fig. 10. For stars of mass $M_{\text {init }} \geq 3.5 M_{\odot}$ with HBB, the yields of oxygen are negative and range from $-0.002 M_{\odot}$ to $-0.012 M_{\odot}$, for masses between 3.5 and $8.5 \mathrm{M}_{\odot}$, respectively.

The yields of nitrogen, $Y_{\mathrm{N}}$, are positive for all the stars. Low-mass stars produce nitrogen via the FDU and the corresponding yields are below $0.005 M_{\odot}$ (see top, right panel of Fig. 16. The N yields of stars experiencing HBB are higher, owing to nitrogen synthesis via $\mathrm{CNO}$ cycling. For these stars we find $0.01 M_{\odot}<Y_{\mathrm{N}}<0.03 M_{\odot}$.

The behaviour of sodium, shown in the right, bottom panel of Fig. 16, is qualitatively similar to nitrogen. In the low mass domain we have $Y_{\mathrm{Na}}<5 \times 10^{-5} M_{\odot}$, whereas for stars with HBB we find a gradual increase with stellar mass, from $Y_{\mathrm{Na}} \sim 3 \times 10^{-4} M_{\odot}$ to $Y_{\mathrm{Na}} \sim 6 \times 10^{-4} M_{\odot}$. This is consistent with the results shown in Fig. 10 .

In the comparison with the models published in Karakas \& Lugaro (2016) we find that the helium yields, shown in Fig. 15, are extremely similar (as discussed in Section 6. The same holds for the results taken from the FRUITY database, published by Cristallo et al. (2015).

The carbon yields of stars of mass $M \geq 3 M_{\odot}$ by Karakas \& Lugaro (2016) are higher than those presented here. This can be clearly seen in the top, left panel of Fig. 16 The largest discrepancy is found for masses $3 M_{\odot}<M<$ $5 M_{\odot}$, for which the present yields are negative whereas the MONASH yields are positive. Similar differences, though of 

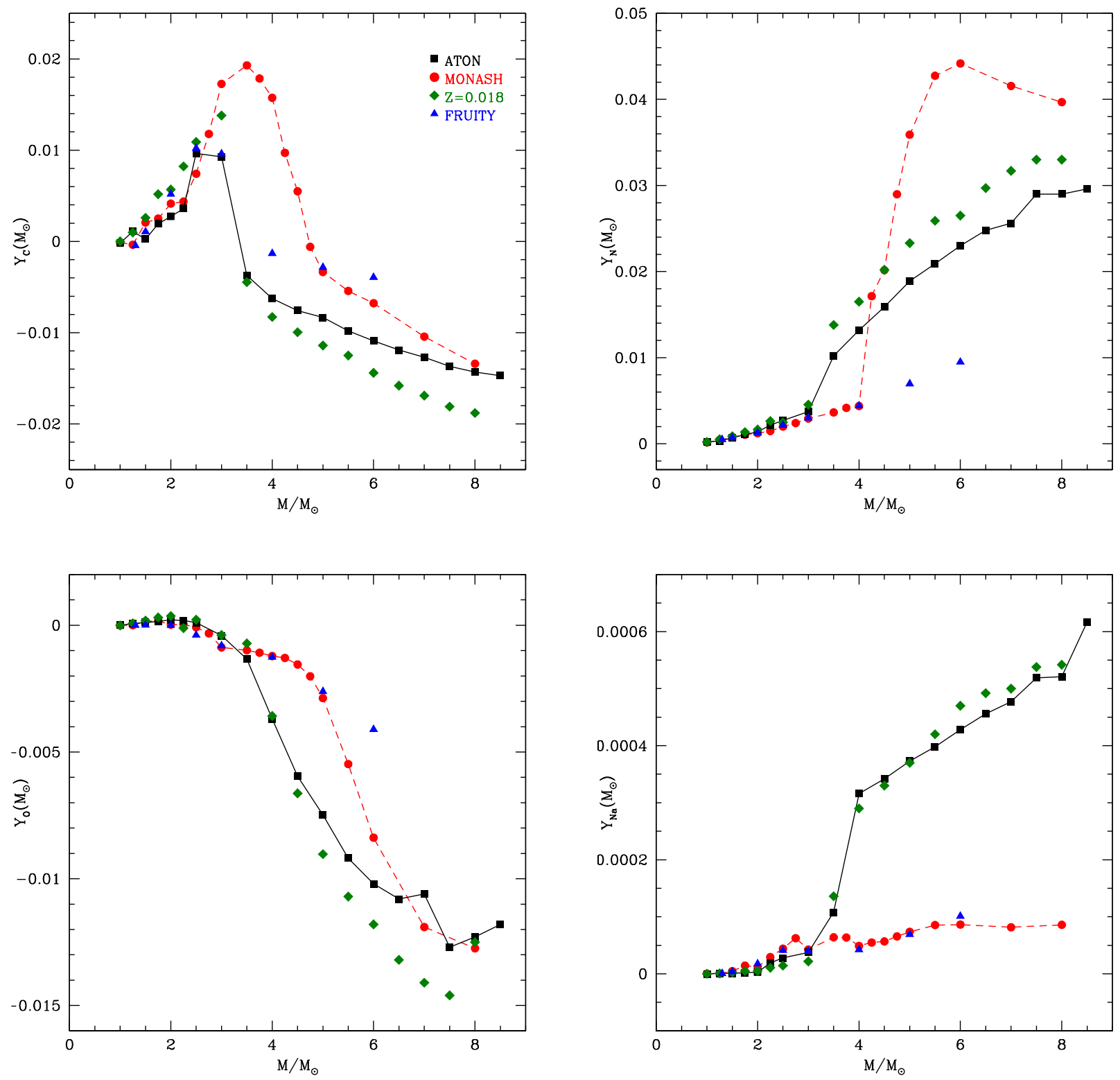

Figure 16. The yields of the CNO elements and of sodium for the AGB models presented here (shown as black squares), compared with the results from Karakas \& Lugaro (2016) (red points), Cristallo et al. (2015) (blue triangles) and Di Criscienzo et al. (2016) (green diamonds). The 4 panels report the yields of ${ }^{12} C$ (top, left panel), ${ }^{14} N$ (top, right), ${ }^{16} \mathrm{O}$ (bottom, left) and sodium (bottom, right).

minor extent, are found when the present yields are compared with results from the FRUITY database. In the massive AGB domain all the sets of carbon yields are negative, but the ATON yields are lower, because the HBB found in these models is stronger and the surface chemistry is affected almost entirely by HBB. Conversely, in the MONASH and FRUITY cases, TDU events increase the surface carbon mass abundance.

The oxygen yields of low-mass stars by Karakas \& Lugaro (2016) and Cristallo et al. (2015) are similar to ours, whereas in the higher mass domain they are higher than those presented here (see Fig. 16). This is a consequence of the different efficiency of HBB, which in turn, is determined by convection modelling. The two lines indicating the ATON and MONASH yields tend to converge towards the most massive models. This is an indication that for large core masses an efficient HBB is found, independently of convection modelling. It is difficult to make a similar comparison to the FRUITY models because the most massive initial masses considered are of $6 M_{\odot}$, lower than the most massive models considered in the ATON or Monash codes.

The results shown in Fig. 16 indicate that a larger pro- 
duction of nitrogen is found by Karakas \& Lugaro (2016). For stars of mass around $6 M_{\odot}$ the MONASH yields are almost a factor of 2 higher than ATON. As discussed in section 7. this is due to TDU events which mix primary carbon from the He-shell to the envelope, which allows the production of primary nitrogen. In the ATON case the nitrogen produced is essentially secondary. The nitrogen FRUITY yields are even smaller than ATON, because HBB is not particularly efficient even in their most massive models.

By looking at Fig. 16 we notice that the largest difference between ATON, MONASH and FRUITY models is found in the sodium yields. In the present models we find some sodium production in the higher mass domain, whereas in the models by Karakas (2014) and Cristallo et al. (2015) the excess of sodium with respect to the initial quantity in the ejecta is negligible. This is consistent with the results shown in Fig. 5

Fig. 15 and 16 also show the comparison between the present yields and those found in Di Criscienzo et al. (2016), which are based on the solar composition by Grevesse \& Sauval (1998). The differences are small and far lower than those introduced by the use of a different description of the convective instability.

\section{DUST FORMATION}

We applied the formulae described in Section 3 to calculate the dust formed in the wind of the models presented here, as well as in the models of solar metallicity by Karakas (2014).

The dust species formed in the wind are primarily determined by the surface $C / O$ ratio. In the wind of oxygen rich stars the majority of the dust produced is in the form of silicates and alumina dust, whereas in carbon stars the main species are carbon and $\mathrm{SiC}$. We will focus our attention on the size of the dust grains formed, on the degree of condensation of the key elements to form dust, and on the total mass of dust produced.

In the previous sections we outlined that low-mass stars with mass in the range $1.5 M_{\odot} \leq M_{\text {init }} \leq 3 M_{\odot}$ become C-rich, whereas more massive stars evolve as oxygen-rich objects. This holds for both the ATON and the MONASH models. Because most of the mass loss in low-mass stars takes place during the C-rich phase (see Fig. 10), we find that most of the dust produced by these stars is under the form of carbonaceous solid particles: solid carbon and $\mathrm{SiC}$.

The evolution of the grain size of the various particles formed in the wind of AGB stars is thoroughly documented in the literature (Ferrarotti \& Gail 2006. Nanni et al. 2013a b, 2014, Ventura et al. 2014) and we do not repeat the details here. We will discuss how dust production works in oxygen-rich and carbon stars, and eventually describe the overall dust formation phenomenon in AGB stars of solar metallicity.

\subsection{Dust formation under HBB conditions}

The top, left panel of Fig. 17 shows the evolution of the grain size of two out of the three silicate species considered (olivine and pyroxene; quartz is not shown, for clarity reasons) and of alumina dust $\left(\mathrm{Al}_{2} \mathrm{O}_{3}\right)$ in the $5 \mathrm{M}_{\odot}$ model presented in Fig. 2 The top, right panel of the same figure shows the condensation factor of silicon and aluminium 5 These results confirm earlier findings (Ventura et al. 2014): a) most of the dust produced is in the form of silicates, the dominant species being olivine, followed by pyroxene; $b$ ) during the HBB phase around $\sim 40 \%$ of silicon is condensed onto dust, whereas the condensation factor of aluminium is higher, around $\sim 80 \%$ (Dell'Agli et al. 2014). In the same figure we see that the typical dust to gas ratio ranges from $10^{-3}$ to $1.5 \times 10^{-3}$, whereas the velocities of the wind fall in the range $15-20 \mathrm{~km} / \mathrm{s}$.

The comparison with the models by Karakas \& Lugaro (2016) highlights that these results are not greatly affected by the input physics used, particularly the treatment of convection and mass loss. While in the previous sections we found significant differences for the chemistry of these stars and the stellar yields, the results in terms of dust production are fairly similar. The explanation is in the dust formation process, in particular the relationship between the growth of dust particles and the dynamics of the wind. ATON models evolve at larger luminosities (see Fig. 2), which based on Eq.2, enhances the effects of radiation pressure on dust particles. This provokes a fast acceleration of the wind (note in the bottom, left panel of Fig. 17 that the MONASH velocities are smaller), which in turn favours the decrease in the gas density (see Eq. 4), hence in the number of molecules available for condensation into dust. The dust formation mechanisms for silicates is self-regulated and this is the reason for the similarity in the results shown in Fig. 17 the findings concerning the production of silicates in the winds of massive AGB stars given here are rather general and independent of AGB modelling.

\subsection{Dust formation in carbon stars}

Fig. 18 shows the results for the dust produced by a $3 M_{\odot}$ star. In this case, for the reasons given above, we focus our attention on $\mathrm{SiC}$ and solid carbon grains.

$\mathrm{SiC}$ is produced efficiently in the wind of carbon stars, owing to the thermodynamic stability of this solid compound (Ferrarotti \& Gail 2006). The typical size of SiC particles is $\sim 0.1 \mu \mathrm{m}$. Indeed we find a saturation condition, such that the residual silicon not bound into the very stable SiS molecules (Ferrarotti \& Gail 2006), i.e. around 55\%, condenses into dust. This corresponds to grain sizes of the order of $0.1 \mu \mathrm{m}$. The saturation process is the reason why the grain size of $\mathrm{SiC}$ grains and the corresponding condensation fraction of silicon remain constant for the whole AGB phase, after the achievement of $\mathrm{C} / \mathrm{O} \geq 1$. Saturation occurs shortly after the beginning of the carbon star phase and is fairly independent of the stellar parameters and the amount of carbon accumulated. This is the reason why the ATON and MONASH results are extremely similar in this regards, as shown in Fig. 18

The dust production process in the winds of carbon stars can be divided into two phases. At the beginning of

\footnotetext{
5 We refer to the condensation factor of a given element as the fraction of the element in the gaseous state that condensed into dust. For these specific cases we refer to the fraction of gaseous silicon condensed into olivine, pyroxene and quartz and to the fraction of gaseous aluminium condensed into $\mathrm{Al}_{2} \mathrm{O}_{3}$.
} 

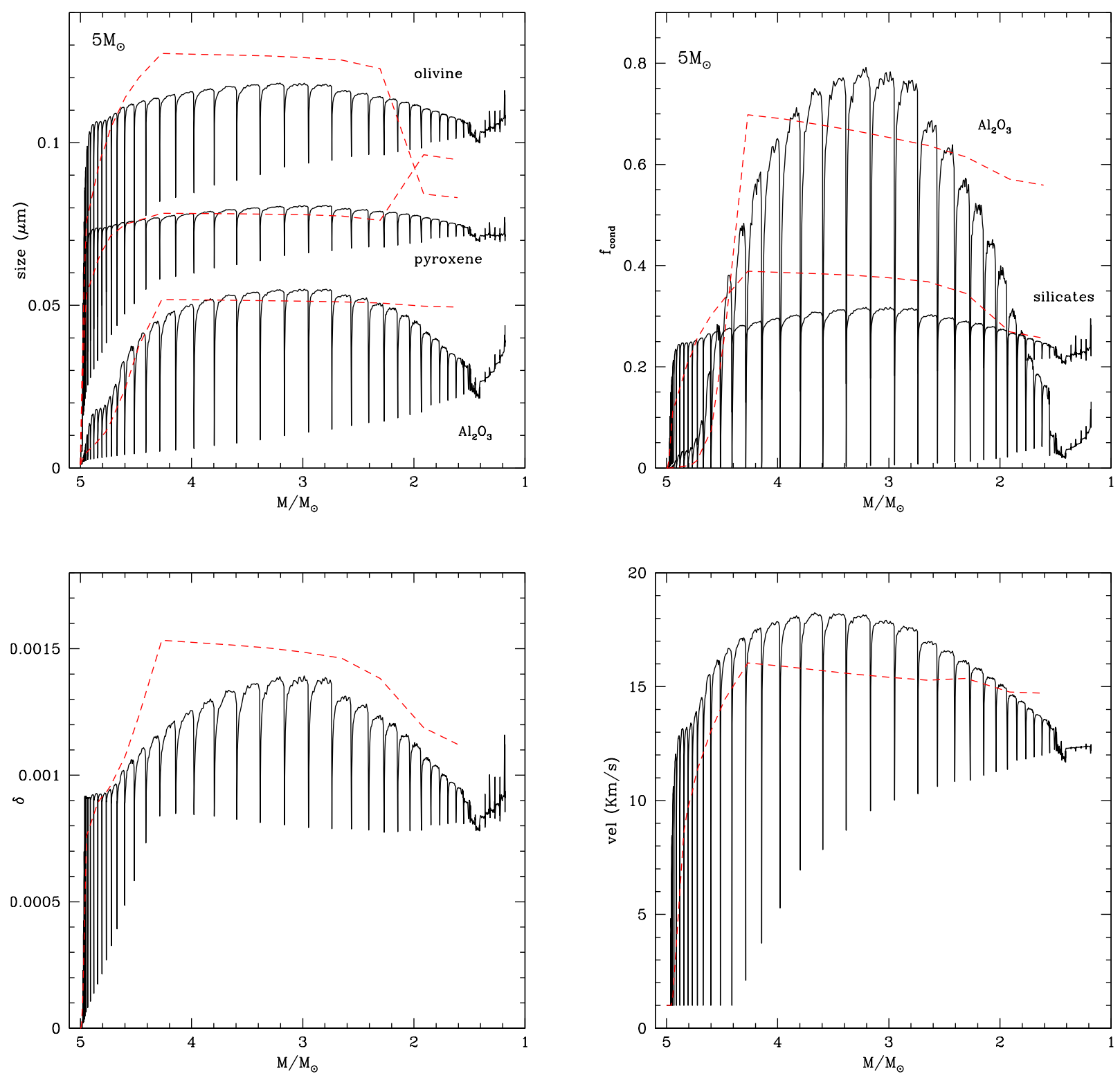

Figure 17. Left: the variation of the grain size of olivine, pyroxene and alumina dust formed in the wind of a $5 M_{\odot}$ during the AGB phase. The results discussed in this work are indicated with a black, solid line and are compared with the results obtained on the basis of the AGB models by Karakas \& Lugaro (2016) are indicated with a red, dashed line. Right: The variation of the fraction of silicon condensed into silicates and of aluminium condensed into $\mathrm{Al}_{2} \mathrm{O}_{3}$ for the same models shown in the left panel.

the C-star phase, when the excess of carbon with respect to oxygen is smaller than the amount of silicon in the envelope, the dominant dust species is SiC. The progressive increase in the surface carbon eventually makes carbon production dominant with respect to $\mathrm{SiC}$. While $\mathrm{SiC}$ particles of the size given above form in an internal region of the circumstellar envelope, solid carbon particles of bigger size are produced in a more external zone. This can be seen in the left panel of Fig. 18, where the size of carbon particles grow bigger and bigger, until reaching dimensions slightly below $0.2 \mu \mathrm{m}$. In the very final phases the fraction of carbon condensed into dust approaches $40 \%$ and the dust-to-gas ratio increases up to $\sim 0.0035$. We reiterate here that these late evolutionary phases, though extremely important for the dust pollution by these stars (as shown in Fig. 18) are extremely short relative to the whole TP-AGB phase, and more importantly, to the duration of the C-star phase.

When comparing Fig. 18 with the results based on MONASH models, we find significant differences for the production of solid carbon. In the MONASH case the fraction of carbon condensed into dust barely exceeds $10 \%$, the maximum size of the carbon grains formed is $0.15 \mu \mathrm{m}$ and the 

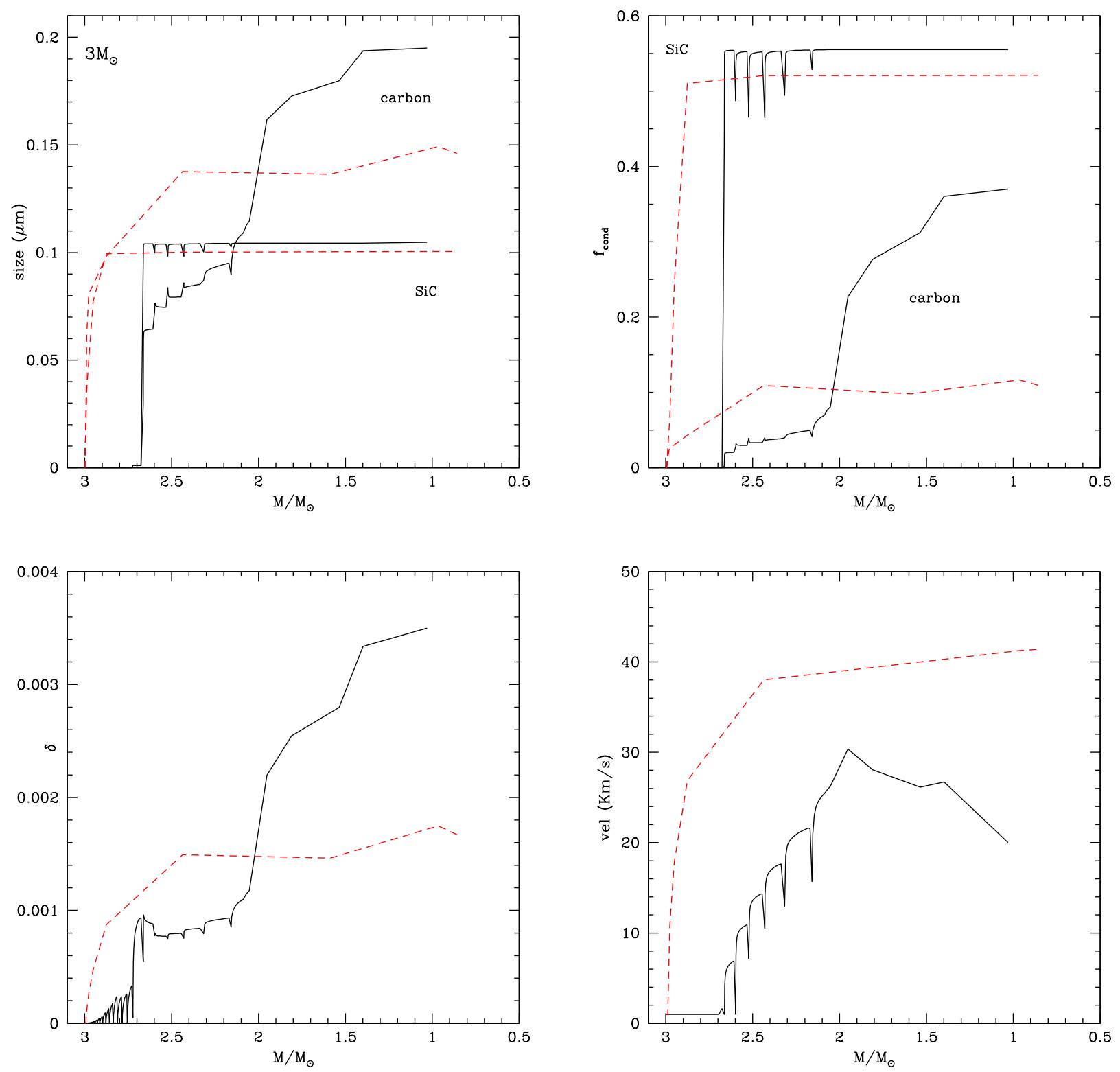

Figure 18. Left: the variation of the grain size of solid carbon and SiC formed in the wind of a $3 M_{\odot}$ during the AGB phase. The results discussed in this work are indicated with a black, solid line and are compared with the results obtained on the basis of the AGB models by Karakas \& Lugaro (2016), indicated with a red, dashed line. Right: The variation of the fraction of gaseous carbon condensed into solid carbon and of silicon condensed into $\mathrm{SiC}$ for the same models shown in the left panel.

dust-to-gas ratio is below 0.002 . The reason for this difference can be deduced based on the results shown in the top, right panel of Fig. 6 and discussed in Section 4.2 In the ATON case, owing to the presence of a narrow and very high overadiabaticity peak, the effective temperatures become extremely cool, which favours very large mass-loss rates, which in turn favours dust production. This does not affect the formation of $\mathrm{SiC}$, as there is no more gaseous silicon available, but strongly enhances the formation of solid carbon. In the MONASH case the effective temperature are hotter, thus no enhanced formation of solid carbon is found. Note that this difference holds despite the fact that the surface carbon is higher in the MONASH model. This is because the growth of carbon grains is much more sensitive to the rate of mass-loss than the surface carbon abundance (Ventura et al. 2016a). 

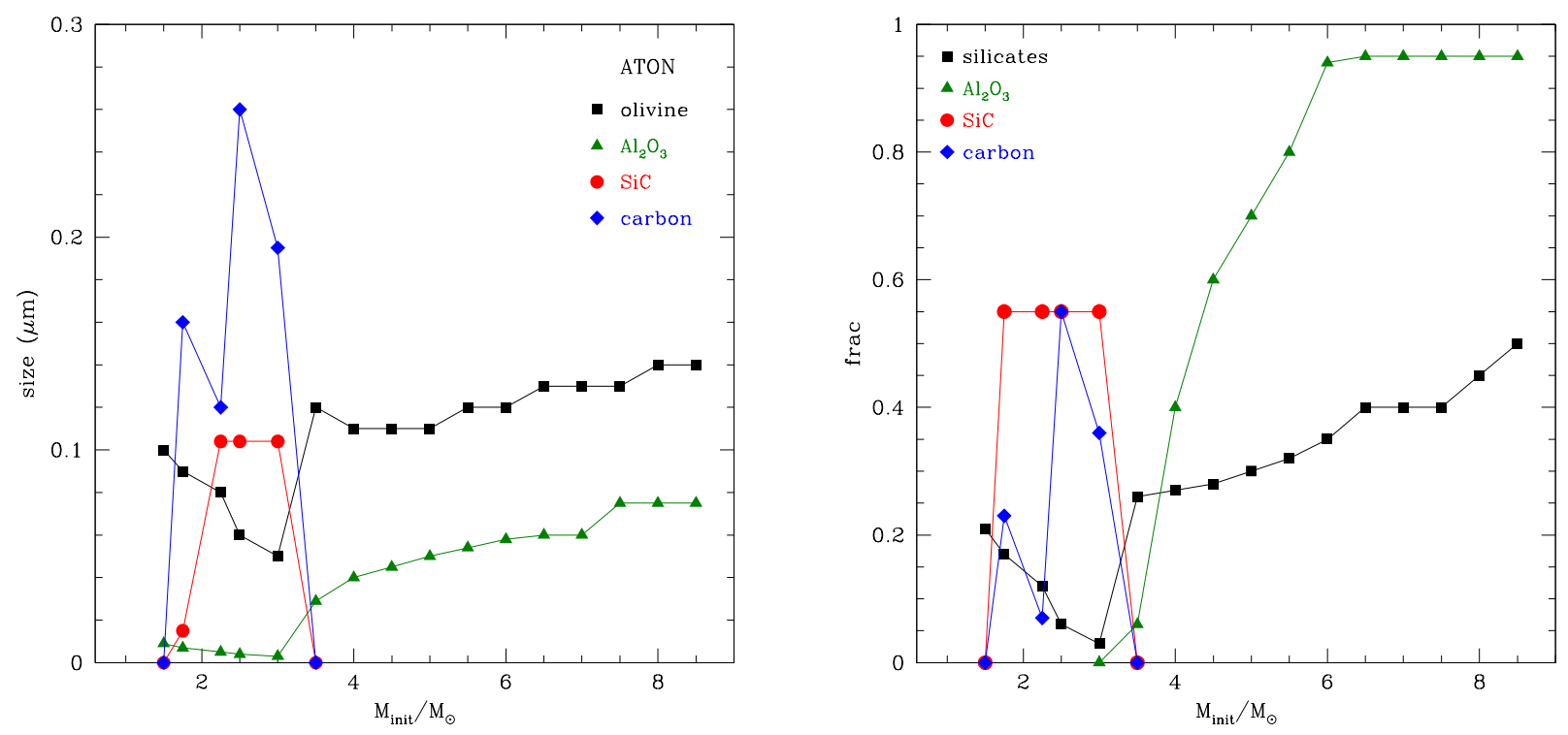

Figure 19. Left: The typical size of dust particles of different species formed in the wind of AGB stars of different mass. The meaning of the various symbols is the following: olivine - black squares; alumina dust - green triangles; SiC - red points; solid carbon - blue diamonds. Right: The fraction of the key elements condensed into solid particles during the AGB phase. The fraction of silicon condensed into silicates and $\mathrm{SiC}$ is indicated, respectively, with black squares and red points; green triangles and blue diamonds indicate the fraction of aluminium involved in $\mathrm{Al}_{2} \mathrm{O}_{3}$ and the fraction of gaseous carbon condensed into carbon grains.

\subsection{The properties of dust particles in solar metallicity AGB stars}

Fig. 19 shows the typical grain size of the various dust particles formed in the wind of stars of different mass during the AGB phase. We also show the fraction of the key species condensed into dust (silicon for silicates and $\mathrm{SiC}$, aluminium for $\mathrm{Al}_{2} \mathrm{O}_{3}$ and carbon).

Low-mass stars with mass $1.75 M_{\odot} \leq M_{\text {init }} \leq 3 M_{\odot}$ produce mainly solid carbon and $\mathrm{SiC}$. Owing to the saturation effect discussed above, the size of the $\mathrm{SiC}$ grains formed and the silicon condensation fraction are $\sim 0.1 \mu \mathrm{m}$ and $55 \%$ respetively, independent of mass. The size of carbon grains span the range $0.1 \mu m<a_{\mathrm{C}}<0.28 \mu m$, while the carbon condensation factor is within $10 \%<f_{\mathrm{C}}<55 \%$. These values depend on the amount of carbon accumulated into the envelope and the rate of mass loss, which change with stellar mass. The carbon grains with the largest size form in the winds of the stars which experience a large number of TDU events and accumulate the largest amount of carbon; as shown in Fig. 10 this occurs for stars of initial mass $2.5-3 M_{\odot}$, which are expected to exhibit extremely large infrared excesses towards the final AGB phases. Note that stars in this mass range have been invoked to reproduce the most obscured sources in the LMC (Dell'Agli et al. 2015a b Ventura et al. 2016a).

Fig. 19 shows that low-mass stars also produce some silicates. These are produced in the evolutionary phases before the achievement of the C-star stage, when the star is still oxygen-rich. For masses around the lower limit to become carbon stars, namely $\sim 1.5 M_{\odot}$, the quantity of silicates produced is higher than $\mathrm{SiC}$ and carbon, because the C-star stage is reached only at the very end of the AGB phase, when a significant fraction of the (oxygen-rich) envelope is already lost.

Interestingly, these stars could be the progenitors of the so-called mixed-chemistry $\mathrm{PNe}$, where both $\mathrm{C}$ - and O-rich IR dust features have been detected simultaneously (Gutenkunst et al. 2008, Perea-Calderón et al. 2009 . Guzman-Ramirez et al. 2011,2014, 2015). These objects show polycyclic aromatic hydrocarbon (PAH) features (Crich) as well as (amorphous/crystalline) silicates features (Orich) and their stellar origin is not understood (see e.g., recent discussions in García-Hernández \& Górny 2014 GarcíaHernández et al. 2016, and references therein). One of the explanations to this mixed-chemistry phenomenon, especially for the case of the PN BD+30 3639 (Guzman-Ramirez et al. 2015), is to invoke a fatal thermal pulse at the very end of the AGB phase (Waters et al. 1998, Perea-Calderón et al. 2009 Guzman-Ramirez et al. 2015). The evolutionary models presented here, show that this mixed-chemistry could naturally arise as part of the evolution of at least some (i.e., those converted to C-rich) low-mass stars. Indeed, GarcíaRojas et al. (2017) have very recently reported precise $\mathrm{C} / \mathrm{O}$ ratios (homogeneously derived) for a sample of 23 mixedchemistry $\mathrm{PNe}$, which combined with the He abundances and N/O ratios, suggest for the first time that some mixedchemistry PNe may be the descendants of very low-mass (M $\left.<1.5 \mathrm{M}_{\odot}\right)$ stars.

In the wind of stars of mass $M \geq 3.5 M_{\odot}$ the only dust species formed are silicates and alumina dust. As shown in the left panel of Fig. 19, the typical size of the grains formed are $0.12-0.14 \mu \mathrm{m}$ and $0.03-0.08 \mu \mathrm{m}$, respectively, for olivine and alumina dust. The fraction of silicon condensed into sil- 


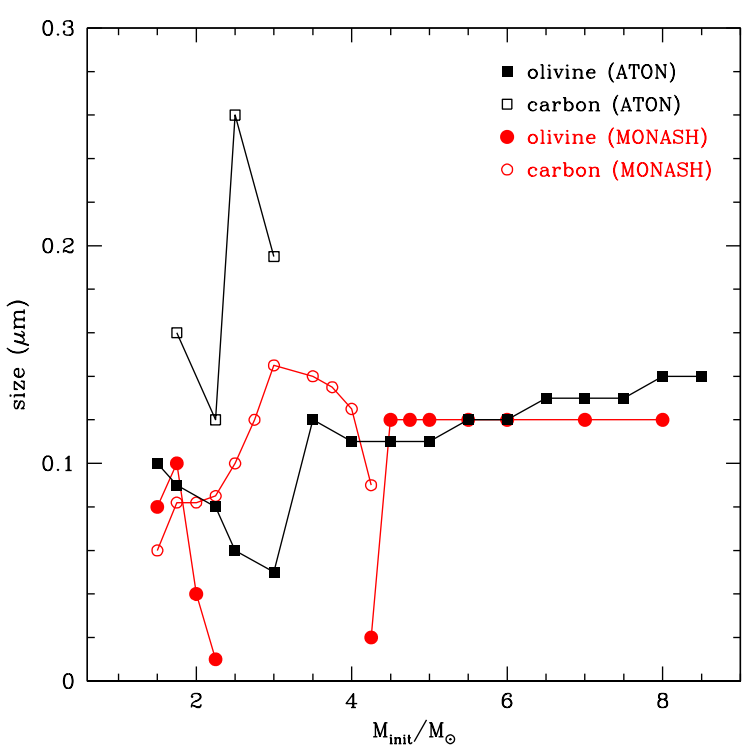

Figure 20. The size of solid carbon (open squares) and olivine (full squares) particles formed in the wind of the AGB models discussed here. Open, red circles and full, red points indicate, respectively, the dimension of solid carbon and olivine grains formed when the MONASH results for the description of the AGB phase are used.

icates ranges from $\sim 25 \%$ to $\sim 50 \%$, whereas for alumina dust the fraction of aluminium condensed into dust ranges from $\sim 10 \%$ to $\sim 95 \%$. The large percentages of gasesous aluminium condensed into dust stems from the large stability of $\mathrm{Al}_{2} \mathrm{O}_{3}$, which forms at temperatures $T \sim 1400 \mathrm{~K}$ in regions of the circumstellar envelope very close to the surface of the star (Dell'Agli et al. 2014). Both the amounts of silicates and of alumina dust formed increase with the mass of the star, because stars of higher mass also experience higher mass-loss rates, which leads to denser winds with a higher number of gaseous molecules available to form dust. This in agreement with previous studies focused on lower metallicity AGB stars (Ventura et al. 2012a b, 2014).

Fig. 20 shows the comparison between the grain sizes of the dust particles found when using the present models and the AGB models by Karakas \& Lugaro (2016). For clarity we only show the most relevant silicate species, olivine, and carbon. Following the discussion above and the results shown in Fig. 18, we know that the size of SiC particles are extremely similar in the two cases.

The dimension of the olivine grains is fairly similar between the ATON and MONASH models. This holds both in the massive AGB domain and for low-mass stars, with $M<2 M_{\odot}$. This is consistent with the results shown in Fig. 18 and with our previous study on the oxygen-rich stars in the LMC with the largest infrared emission (Ventura et al. 2015a). The only difference holds in the range of mass $2 M_{\odot}<M<4 M_{\odot}$, where we find some silicate production in the present models, whereas a negligible amount of silicates form in the MONASH case. This is because in the latter case the achievement of the C-star stage occurs when

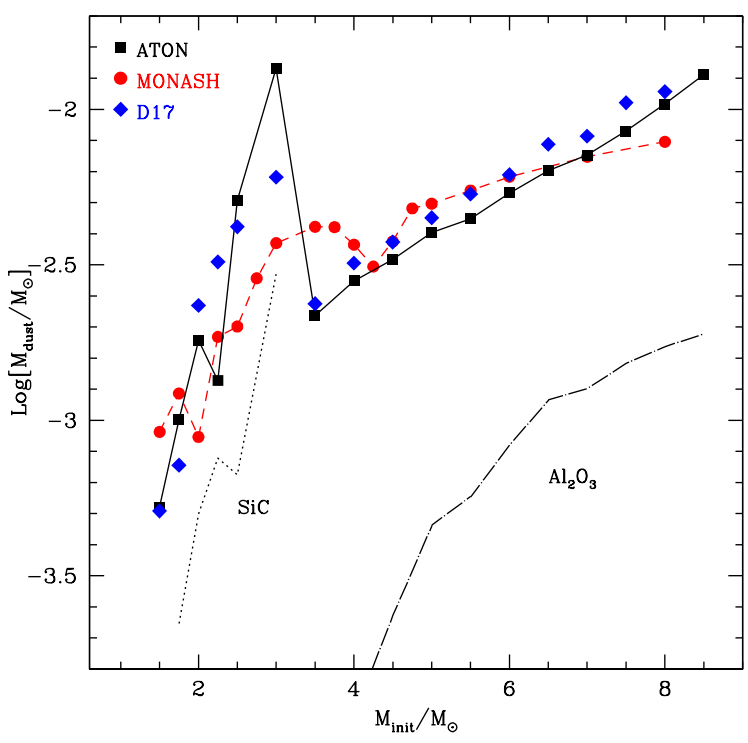

Figure 21. The total dust mass produced by solar metallicity, AGB models is shown as a function of the initial mass of the star and indicated with black, full squares. The results based on the MONASH models for the AGB evolution are indicated with full, red points, whereas the results from D17 are shown as blued diamonds.

only a tiny fraction of the envelope was lost, thus all the dust formed and ejected into the interstellar medium is under the form of carbonaceous dust.

The ATON and MONASH results are similar for carbon stars of mass $M \leq 2 M_{\odot}$, whereas they differ for $M \sim$ $2-2.5 M_{\odot}$. In the present models we find a much larger formation of solid carbon particles, which reach sizes in the range $0.2 \mu m<a_{C}<0.28 \mu \mathrm{m}$. Conversely, when using the MONASH models, we find carbon grain dimensions below $0.15 \mu \mathrm{m}$. This is the only relevant difference found among the two sets of models, which has been extensively discussed in Section 9.2

\subsection{The overall dust mass budget by AGB stars}

We conclude this analysis with the discussion on the dust mass produced by AGB stars of solar metallicity. In Fig. 21 we show the total dust mass produced by stars of different mass during the AGB phase. The values of the dust mass of the individual species formed are reported in Table 3 . the total dust mass produced is indicated in the last column of the Table. As discussed previously, most of the dust produced by low-mass stars is solid carbon, whereas for massive AGB stars the majority of the dust formed are silicates. The mass of $\mathrm{SiC}$ and alumina dust produced is significantly smaller than the mass of carbon and silicates, and are shown separately in Fig. 21

Stars with mass in the range $3.5 M_{\odot}<M<8.5 M_{\odot}$ produce dust masses in the range $2 \times 10^{-3} M_{\odot}<M<$ $10^{-2} M_{\odot}$. This dust is mainly silicates: the contribution of alumina dust is below $\sim 20 \%$ (see Fig. 21). Low-mass stars 
Table 3. Dust masses (in solar masses) produced by solar metallicity AGB stars.

\begin{tabular}{ccccccccc}
\hline \hline $\mathrm{M}$ & $M_{\mathrm{ol}}$ & $M_{\mathrm{py}}$ & $M_{\mathrm{qy}}$ & $M_{A l_{2} \mathrm{O}_{3}}$ & $M_{\mathrm{SiC}}$ & $M_{\mathrm{C}}$ & $M_{\text {ir }}$ & $M_{\text {dust }}$ \\
\hline 1.50 & $1.05 \mathrm{E}-04$ & $3.67 \mathrm{E}-05$ & $1.13 \mathrm{E}-05$ & $1.28 \mathrm{E}-07$ & $0.00 \mathrm{E}+00$ & $0.00 \mathrm{E}+00$ & $3.73 \mathrm{E}-04$ & $5.26 \mathrm{E}-04$ \\
1.75 & $8.00 \mathrm{E}-05$ & $2.82 \mathrm{E}-05$ & $9.21 \mathrm{E}-06$ & $7.28 \mathrm{E}-08$ & $2.22 \mathrm{E}-04$ & $5.48 \mathrm{E}-04$ & $1.19 \mathrm{E}-04$ & $1.01 \mathrm{E}-03$ \\
2.00 & $2.59 \mathrm{E}-05$ & $9.42 \mathrm{E}-06$ & $3.94 \mathrm{E}-06$ & $2.64 \mathrm{E}-08$ & $5.00 \mathrm{E}-04$ & $0.00 \mathrm{E}+00$ & $1.27 \mathrm{E}-03$ & $1.81 \mathrm{E}-03$ \\
2.25 & $2.33 \mathrm{E}-05$ & $1.12 \mathrm{E}-05$ & $4.38 \mathrm{E}-06$ & $1.25 \mathrm{E}-08$ & $7.55 \mathrm{E}-04$ & $2.97 \mathrm{E}-04$ & $2.50 \mathrm{E}-04$ & $1.34 \mathrm{E}-03$ \\
2.50 & $6.70 \mathrm{E}-06$ & $2.65 \mathrm{E}-06$ & $1.37 \mathrm{E}-06$ & $6.19 \mathrm{E}-09$ & $6.68 \mathrm{E}-04$ & $4.30 \mathrm{E}-03$ & $1.22 \mathrm{E}-04$ & $5.10 \mathrm{E}-03$ \\
3.00 & $2.77 \mathrm{E}-06$ & $1.21 \mathrm{E}-06$ & $5.21 \mathrm{E}-07$ & $3.94 \mathrm{E}-09$ & $2.95 \mathrm{E}-03$ & $1.05 \mathrm{E}-02$ & $8.52 \mathrm{E}-05$ & $1.35 \mathrm{E}-02$ \\
3.50 & $1.43 \mathrm{E}-03$ & $4.41 \mathrm{E}-04$ & $5.63 \mathrm{E}-05$ & $1.90 \mathrm{E}-05$ & $0.00 \mathrm{E}+00$ & $0.00 \mathrm{E}+00$ & $2.54 \mathrm{E}-04$ & $2.19 \mathrm{E}-03$ \\
4.00 & $1.99 \mathrm{E}-03$ & $6.12 \mathrm{E}-04$ & $5.48 \mathrm{E}-05$ & $1.13 \mathrm{E}-04$ & $0.00 \mathrm{E}+00$ & $0.00 \mathrm{E}+00$ & $1.45 \mathrm{E}-04$ & $2.92 \mathrm{E}-03$ \\
4.50 & $2.41 \mathrm{E}-03$ & $7.35 \mathrm{E}-04$ & $4.81 \mathrm{E}-05$ & $2.36 \mathrm{E}-04$ & $0.00 \mathrm{E}+00$ & $0.00 \mathrm{E}+00$ & $9.83 \mathrm{E}-05$ & $3.52 \mathrm{E}-03$ \\
5.00 & $2.96 \mathrm{E}-03$ & $9.09 \mathrm{E}-04$ & $5.18 \mathrm{E}-05$ & $4.59 \mathrm{E}-04$ & $0.00 \mathrm{E}+00$ & $0.00 \mathrm{E}+00$ & $9.75 \mathrm{E}-05$ & $4.48 \mathrm{E}-03$ \\
5.50 & $3.33 \mathrm{E}-03$ & $9.98 \mathrm{E}-04$ & $4.61 \mathrm{E}-05$ & $5.67 \mathrm{E}-04$ & $0.00 \mathrm{E}+00$ & $0.00 \mathrm{E}+00$ & $6.78 \mathrm{E}-05$ & $5.01 \mathrm{E}-03$ \\
6.00 & $4.09 \mathrm{E}-03$ & $1.19 \mathrm{E}-03$ & $4.11 \mathrm{E}-05$ & $8.34 \mathrm{E}-04$ & $0.00 \mathrm{E}+00$ & $0.00 \mathrm{E}+00$ & $5.92 \mathrm{E}-05$ & $6.21 \mathrm{E}-03$ \\
6.50 & $4.83 \mathrm{E}-03$ & $1.41 \mathrm{E}-03$ & $4.17 \mathrm{E}-05$ & $1.16 \mathrm{E}-03$ & $0.00 \mathrm{E}+00$ & $0.00 \mathrm{E}+00$ & $6.29 \mathrm{E}-05$ & $7.50 \mathrm{E}-03$ \\
7.00 & $5.47 \mathrm{E}-03$ & $1.55 \mathrm{E}-03$ & $4.08 \mathrm{E}-05$ & $1.26 \mathrm{E}-03$ & $0.00 \mathrm{E}+00$ & $0.00 \mathrm{E}+00$ & $6.58 \mathrm{E}-05$ & $8.39 \mathrm{E}-03$ \\
7.50 & $6.55 \mathrm{E}-03$ & $1.85 \mathrm{E}-03$ & $3.73 \mathrm{E}-05$ & $1.52 \mathrm{E}-03$ & $0.00 \mathrm{E}+00$ & $0.00 \mathrm{E}+00$ & $7.70 \mathrm{E}-05$ & $1.00 \mathrm{E}-02$ \\
8.00 & $8.13 \mathrm{E}-03$ & $2.16 \mathrm{E}-03$ & $3.10 \mathrm{E}-05$ & $1.72 \mathrm{E}-03$ & $0.00 \mathrm{E}+00$ & $0.00 \mathrm{E}+00$ & $1.06 \mathrm{E}-04$ & $1.21 \mathrm{E}-02$ \\
8.50 & $1.01 \mathrm{E}-02$ & $2.58 \mathrm{E}-03$ & $2.40 \mathrm{E}-05$ & $1.89 \mathrm{E}-03$ & $0.00 \mathrm{E}+00$ & $0.00 \mathrm{E}+00$ & $1.24 \mathrm{E}-04$ & $1.48 \mathrm{E}-02$ \\
\hline
\end{tabular}

in the range $1.75 M_{\odot} \leq M \leq 3 M_{\odot}$ produce carbonaceous particles, in quantities above $10^{-3} M_{\odot}$. We note the peak of $\sim 10^{-2} M_{\odot}$ in the dust mass produced for stellar masses $2.5-3 M_{\odot}$, due to the abundant production of solid carbon in the wind of this stars. The contribution of $\mathrm{SiC}$ to the total dust produced ranges from $\sim 15 \%$ to $\sim 30 \%$.

These results are in substantial agreement with the results published by D17, indicating that the details of the solar mixture adopted has a minor effect on the dust mass expected.

The comparison with the results based on the AGB models by Karakas \& Lugaro (2016) shows that the results in the massive AGB domain are extremely similar. In the low-mass domain the amount of carbon dust expected when using the MONASH models is a factor $\sim 3$ smaller, owing to the differences in convection modelling. Note that this dissimilarity is found only in a limited range of mass, namely for $2.5-3 M_{\odot}$ stars.

\section{THE ROLE OF METALLICITY ON THE EVOLUTIONARY PROPERTIES OF AGB STARS}

In our previous studies we used AGB models of sub-solar metallicity $\left(Z=4,8 \times 10^{-3}\right)$ to interpret the evolved stars in the MC with the largest infrared excess (Ventura et al. 2015a, 2016a). The comparison of theoretical results with the observational evidence is more tricky in the present case, because the poor knowledge of the distances of Galactic sources prevents a straightforward interpretation of the currently available observations of solar metallicity, AGB stars.

Gaia and future space missions are likely alter this framework, with highly accurate determination of the parallaxes of several AGB sources and the availability of highquality data. To be prepared for the interpretation of these results we discuss the differences between the present models and the cases discussed in Ventura et al. (2015a, 2016a).

On the physical side, if we focus on stars with mass above $\sim 3.5 M_{\odot}$, we find that the HBB experienced at the bottom of the convective mantle is weaker in the present models than in Ventura et al. (2015a, 2016a). This result is in agreement with previous studies, focused on the sensitivity of the strength of HBB to the metallicity (Ventura et al. 2013). Fig. 22 shows the temperature at the base of the envelope of stars of various initial mass and metallicity. The differences between the models discussed here and their $Z=$ $4 \times 10^{-3}$ counterparts reach $\delta T \sim 15 \mathrm{MK}$ in the $M>6 M_{\odot}$ domain. Given the steep sensitivity of the nuclear proton capture cross sections for temperatures in the range $70-$ $100 \mathrm{MK}$, this reflects into a much more advanced p-capture nucleosynthesis in lower metallicity stars.

Turning to the low-mass regime, all the stars with initial mass $1 M_{\odot}<M<3 M_{\odot}$ become carbon stars during the AGB evolution. In the present models the C-star condition is reached in a more advanced phase compared to the models used in Ventura et al. (2015a) and Ventura et al. (2016a), owing to the initial higher oxygen content, which delays the $C / O>1$ condition. As shown in the right panel of Fig. 22 , the time fraction of the phase during which these stars are Crich is significantly shorter in the solar case, ranging from $\sim$ $2 \%$ to $\sim 15 \%$, compared to the lower metallicity chemistries. We expect to detect a smaller fraction of carbon stars in the solar metallicity environment than in the MC.

The differences outlined above affect the evolution of the surface chemical composition of AGB stars. To show this, we report in Fig. 23 the final surface abundances of oxygen and nitrogen, in terms of the ratio with respect to the initial values.

In the solar case, indicated by black squares, the surface oxygen of low-mass stars (reported in the lower, right region of the diagram) remains practically unchanged during the AGB life, because the initial oxygen is too large to be meaningfully affected by TDU. Conversely, for subsolar metallicities, the amount of oxygen transported to the surface regions via repeated TDU events is sufficient to determine a significant variation, with a maximum increase of the order of a factor $\sim 3$, for the $Z=4 \times 10^{-3}, 2 M_{\odot}$ model.

In the three lines shown in Fig. 23 we note the transition to the massive domain, in the sudden increase in the surface 

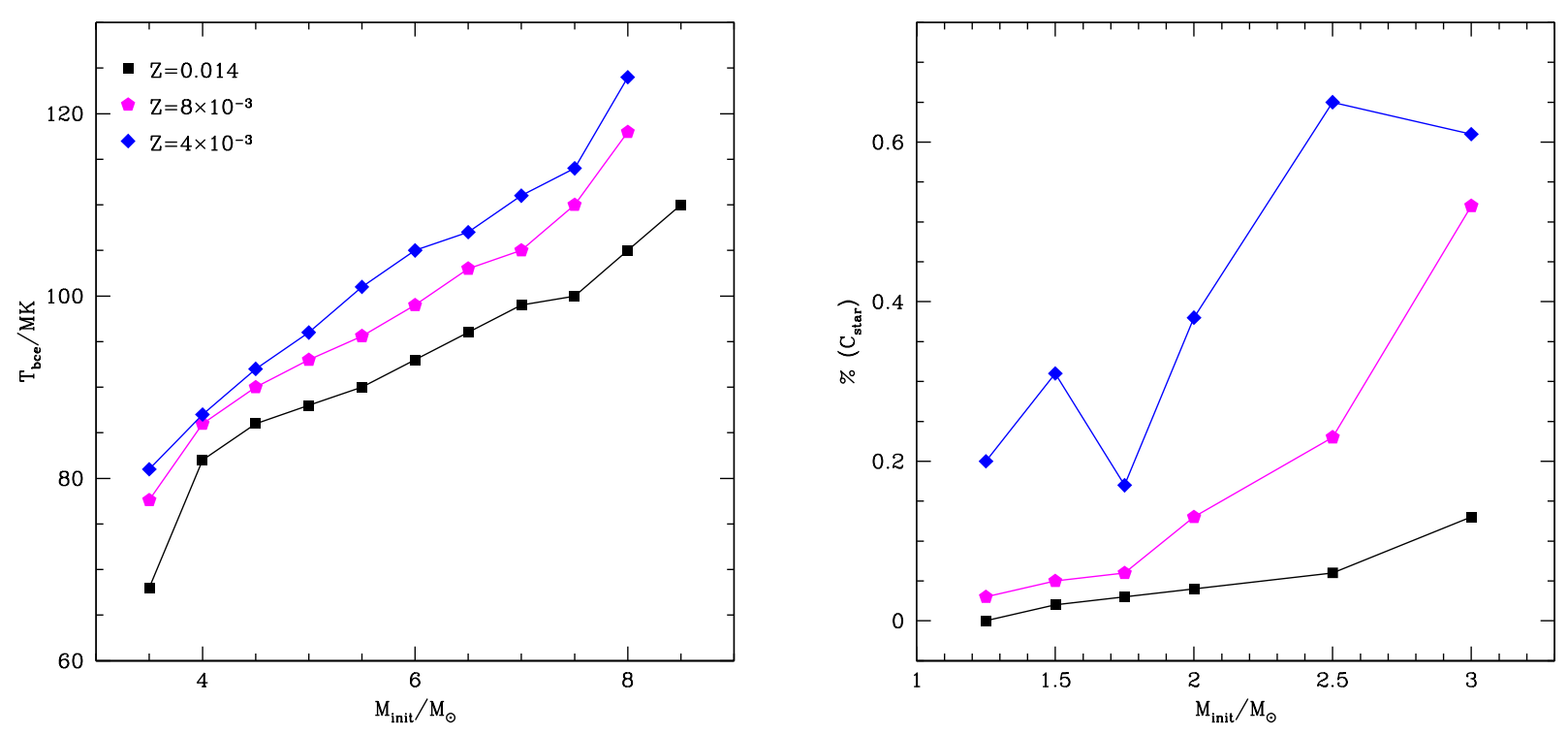

Figure 22. Left: The temperature at the base of the convective envelope experienced by the solar metallicity models presented in this work (black squares), compared with their counterparts of metallicity $Z=4 \times 10^{-3}$ (blue diamonds) and $Z=8 \times 10^{-3}$ (magenta pentagons). Right: The time fraction of the C-star phase in relation to the total duration of the AGB phase of low-mass, AGB models of different metallicity. The meaning of the symbols is the same as in the left panel.

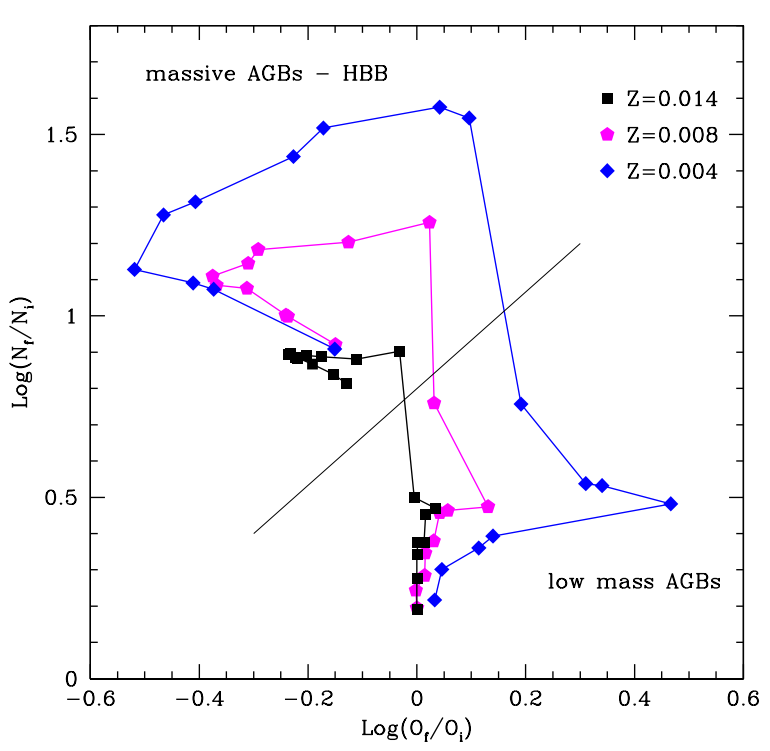

Figure 23. The final surface chemical composition of the same models presented in Fig. 22, in terms of the variation of the oxygen (reported on the abscissa) and nitrogen (y-axis) mass fraction, compared to the quantities initially present in the star. The three lines connect models of the same metallicity, with the mass increasing counter-clock wise. The diagonal line marks the distinction between low-mass AGB stars, whose chemical composition is determined by TDU, and massive AGB stars, affected by HBB. nitrogen, a clear signature of the effects of HBB, as also the decrease in the surface oxygen abundance. The latter effect is more evident in lower metallicity models, because of the more advanced nucleosynthesis experienced, according to the discussion above and the results shown in the left panel of Fig. 22

The differences in the physical behaviour and in the variation of the surface chemical composition of AGB stars of different metallicity reflect into the dust production. Fig. 24 shows the typical size of solid carbon (for stars of mass $\left.M \leq 3 M_{\odot}\right)$ and olivine grains $\left(M>3 M_{\odot}\right)$. We focus on these two compounds because they are the dust species formed in the largest quantities in C-rich and O-rich gas, respectively, and, more important, because these are the solid particles providing the most important contribution to the degree of obscuration of the star (Ferrarotti \& Gail 2006).

The size of the olivine grains formed is bigger in the solar metallicity models compared to their lower- $Z$ counterparts, owing to the larger amount of silicon available. This trend, though limited to smaller metallicities, was discussed in Ventura et al. (2014). For what attains the carbonaceous particles formed, the largest dimensions reached, of the order of $\sim 0.27 \mu \mathrm{m}$, are substantially independent of metallicity: this is because the carbon dredged-up during the TDU events is of primary origin and is almost independent of the metallicity of the stars. On the other hand, in the solar case only AGB stars of mass $2.5-3 M_{\odot}$ produce significant quantities of carbon dust; this is due to the later achievement of the C-star stage, as discussed earlier in this section (see the right panel of Fig. 22. 


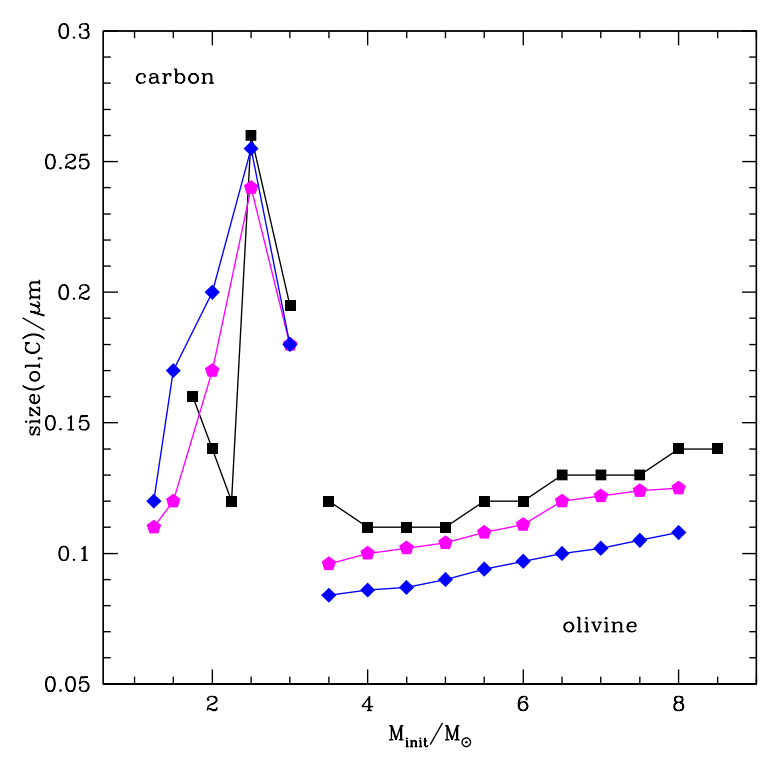

Figure 24. The typical size of the dust particles formed in the wind of AGB stars of different mass and metallicity. The colour coding is the same as in Fig. 22 For low mass stars we give the size of solid carbon grains, whereas for massive AGB stars we report the size of olivine particles.

\section{CONCLUSIONS}

We present updated models of stars with masses between $1 M_{\odot}-8.5 M_{\odot}$, evolved through the AGB phase. The chemical composition of the models, with metallicity $Z=0.014$, reflects the chemistry of the Sun, thus it is suitable to interpret the observations of Galactic AGB stars in the solar neighborhood and beyond. To evaluate how the various results presented are affected by the description of the input physics adopted, we compare the present results with those obtained with the MONASH evolution code, which are based on different input physics.

On the physical side, the behaviour of the models is mainly determined by the mass of the star, with an abrupt transition occurring around $\sim 3.5 M_{\odot}$. Lower mass stars experience TDU, which produces a gradual enrichment in carbon of their surface layers. Stars with initial mass $1.5 M_{\odot} \leq$ $M \leq 3 M_{\odot}$ reach the $\mathrm{C}$-star stage with $1.2<\mathrm{C} / \mathrm{O}$ ratios $<3$ at the tip of the AGB. While the TP-AGB phase of these stars lasts between $1 \mathrm{Myr}$ and $5 \mathrm{Myr}$, the duration of the C-star phase is below $\sim 15 \%$ of the overall TP-AGB evolution. The ejecta are enriched in carbon and show a modest increase in the nitrogen content.

Stars of mass above $3.5 M_{\odot}$ experience HBB at the base of the convective envelope. Their evolution times, decreasing with the mass of the star, are in the range $3 \times 10^{4} y r<$ $\tau_{T P-A G B}<3 \times 10^{5} \mathrm{yr}$. Their surface chemistry shows the signature of $\mathrm{HBB}$ nucleosynthesis, with a significant depletion of carbon and a considerable production of nitrogen; oxygen and heavier species are almost unchanged at the present metallicity. The final $\mathrm{C} / \mathrm{O} \sim 0.05$ and $\mathrm{N} / \mathrm{O} \sim 1.8$ ratios are almost independent of the initial mass. The pollution from these stars is made up of C-poor gas, significantly enriched in nitrogen.

Dust production by AGB stars is also very sensitive to their initial stellar mass. Stars of mass $1.5 M_{\odot} \leq M \leq$ $3 M_{\odot}$ form mainly solid carbon particles, of size $0.1-0.2 \mu \mathrm{m}$; the solid carbon mass produced during the whole stellar life ranges from $10^{-3} M_{\odot}$ to $2 \times 10^{-2} M_{\odot}$, according to the initial mass of the star. The second most abundant dust species formed in these stars is silicon carbide: $\mathrm{SiC}$ grains reach typical size of $\sim 0.1 \mu \mathrm{m}$, whereas the total mass of $\mathrm{SiC}$ dust produced is in the range $3 \times 10^{-4}-3 \times 10^{-3} M_{\odot}$.

Massive AGB stars experiencing $\mathrm{HBB}$ form silicates and alumina dust. The amount of silicates produced increases with the initial mass of the star, ranging from $2 \times 10^{-3} M_{\odot}$ to $10^{-2} M_{\odot}$; the typical size of silicate particles are $0.1-$ $0.15 \mu \mathrm{m}$. Owing to the small content of aluminium compared to silicon, the mass of alumina dust produced is $5-10$ times smaller than the mass of silicates, whereas the dimension of alumina dust grains is about half of that of silicates.

The comparison between the present results with those obtained with the MONASH code outlines strong similarities in the results of low-mass stars, in terms of the evolution of luminosity and core mass, the variation of the surface chemical composition and the duration of the whole AGB phase. Use of either ATON or MONASH models would lead to similar conclusions in the interpretation of observations of Galactic low-mass AGB stars with solar or nearly solar metallicity.

The description of the AGB evolution of stars of mass above $3.5 M_{\odot}$ is more uncertain, because the results obtained depend critically on the convective model adopted, which affects directly the overall energy release, the growth rate of the core mass and the variation of the surface chemistry. In the near future, observational results from ongoing space missions will likely allow a better understanding of the main properties of these stars.

The predictions concerning silicates, alumina dust and SiC produced by AGB stars of solar metallicity are also similar, allowing a model-independent interpretation of IR observations of of both O- and C-rich, dust-enshrouded stars, and the determination of the global dust budget from AGB stars, particularly in galaxies with recent star formation. The amount of carbon dust produced is still affected by significant uncertainties, primarily associated to the description of overadiabatic convection on the outermost regions of C-rich stars.

\section{ACKNOWLEDGMENTS}

DAGH was funded by the Ramón y Cajal fellowship number RYC-2013-14182. DAGH and FD acknowledge support provided by the Spanish Ministry of Economy and Competitiveness (MINECO) under grant AYA-2014-58082-P. AIK acknowledge support provided by the Australian Research Council (DP170100521 and FT110100475).

\section{REFERENCES}

Abia, C., Busso, M., Gallino, R., Domnguez, I., Straniero, O., Isern, J. 2001, ApJ, 559, 1117 
Abia, C., Hedrosa, R. P., Domínguez, I., Straniero, O. 2017, A\&A, 599, A39

Asplund M., Grevesse N., Sauval A. J., Scott P., 2009, ARA\&A, 47, 481

Blanton, M. R., Bershady, M. A., Abolfathi, B. et al. 2017, AJ, 154, 28

Blöcker T., 1995, A\&A, 297, 727

Blöcker T., Schöenberner D., 1991, A\&A, 244, L43

Boothroyd A. I, Sachmann I.-J., 1999, ApJ, 510, 232

Cameron A. G. W., Fowler W. A. 1971, ApJ, 164, 111

Canuto V. M. C., Mazzitelli I., 1991, ApJ, 370, 295

Cristallo S., Straniero O., Piersanti L., Gobrecht D. 2015, ApJS, 219, 40

Delgado-Inglada, G., Morisset, C., \& Stasińska, G. 2014, MNRAS, 440, 536

Dell'Agli F., Ventura P., García-Hernández D. A., Schneider R., Di Criscienzo M., Brocato E., D'Antona F., Rossi C., 2014, MNRAS, 442, L38

Dell'Agli F., Ventura P., Schneider R., Di Criscienzo M., García-Hernández D. A., Rossi C., Brocato E. 2015a, MNRAS, 447, 2992

Dell'Agli F., García-Hernández D. A., Ventura P., Schneider R., Di Criscienzo M., Rossi C. 2015b, MNRAS, 454, 4235

Dell'Agli F., García-Hernández D. A., Schneider R., et al. 2017, MNRAS, 467, 4431

De Smedt, K., Van Winckel, H., Kamath, D., Siess, L., Goriely, S., Karakas, A. I., Manick, R. 2016, A\&A, 587, A6

Di Criscienzo M., Dell'Agli F., Ventura P., Schneider R., Valiante R., La Franca F., Rossi C., Gallerani S., Maiolino, R., 2013, MNRAS, 433, 313

Di Criscienzo M., Ventura P., García-Hernández D. A., et al. 2016, MNRAS, 462, 395

Ferrarotti A. D., Gail H. P., 2001, A\&A, 371, 133

Ferrarotti A. D., Gail H. P., 2002, A\&A, 382, 256

Ferrarotti A. D., Gail H. P., 2006, A\&A, 553, 576

Fishlock C. K., Karakas A. I., Lugaro M., Yong, D. 2014, ApJ, 797, 44

Frost C. A., Lattanzio J. C. 1996, ApJ, 473, 383

Gail H.-P., \& Sedlmayr E. 1999, A\&A, 347, 594

García-Hernández D. A., García-Lario P., Plez B., et al. 2006, Science, 314, 1751

García-Hernández D. A., García-Lario P., Plez B., Manchado A., D'Antona F., Lub J., Habing H. 2007, A\&A, 462,711

García-Hernández D. A., Manchado A., Lambert D. L., et al. 2009, ApJL, 705, L31

García-Hernández D. A., Zamora O., Yagüe A., Uttenthaler S., Karakas A. I., Lugaro M., Ventura P., Lambert D. L. 2013, A\&A, 555, L3

García-Hernández D. A. \& Górny, S. K. 2014, A\&A, 567, A12

García-Hernández, D. A., Ventura, P., Delgado-Inglada, G., Dell'Agli, F., Di Criscienzo, M., Yagüe, A. 2016, MNRAS, 461, 542

García-Rojas, J., Delgado-Inglada, G., García-Hernández D. A., Dell'Agli, F., Lugaro, M., Karakas, A. I. 2017, MNRAS (submitted)

Grevesse N., Sauval A. J 1998, SSrv, 85, 161

Groenewegen, M. A. T., \& Whitelock, P. A. 1996, MNRAS, 281,1347
Gutenkunst S., Bernard-Salas J., Pottasch S. R., Sloan G. C., Houck J. R., 2008, ApJ, 680, 1206-1221

Guzman-Ramirez L., Zijlstra A. A., Níchuimín R., Gesicki K., Lagadec E., Millar T. J., Woods P. M., 2011, MNRAS, 414, 1667

Guzman-Ramirez L., Lagadec E., Jones D., Zijlstra A. A., Gesicki K., 2014, MNRAS, 441, 364

Guzman-Ramirez L., et al., 2015, MNRAS, 451, L1

Hale S. E., Champagne A. E., Iliadis C., Hansper V. Y., Powell D. C., Blackmon J. C. 2002, Phys. Rev. C, 65, 015801

Hedrosa, R. P., Abia, C., Busso, M., Cristallo, S., Domínguez, I., Palmerini, S., Plez, B., Straniero, O. 2013, ApJL, 768, L11

Herwig F. 2005, ARA\&A, 43, 435

Iglesias C. A., Rogers F. J. 1996, ApJ, 464, 943

Izzard R. G., Lugaro M., Karakas A. I., Iliadis C., van Raai, M. 2007, A\&A, 466, 641

Jones O. C., Meixner M., Justtanont K., Glasse A. 2017, ApJ, 841, 15

Kalirai S. J., Marigo P., Tremblay P. E. 2014, ApJ, 782, 17

Karakas A. I. 2014, MNRAS, 445, 347

Karakas A. I., Lattanzio J. C. 2014, PASA, 31, e030

Karakas A. I., Lugaro M. 2016, arXiv:1604.02178

Kobayashi C., Karakas A. I., Umeda H. 2011, MNRAS, 414,3231

Lattanzio J. C. 1986, ApJ, 311, 708

Maraston C., Daddi E., Renzini A., et al. 2006, ApJ, 652, 85

Marigo P. 2002, A\&A, 387, 507

Marigo P., Aringer B., 2009, A\&A, 508, 1538

Nanni A., Bressan A., Marigo P., Girardi L., 2013a, MNRAS, 434, 488

Nanni A., Bressan A., Marigo P., Girardi L., 2013b, MNRAS, 434, 2390

Nanni A. Bressan A. Marigo P. Girardi L., 2014, MNRAS, 438, 2328

Paczyński, B. 1970, Acta Astr., 20, 47

Perea-Calderón, J. V., García-Hernández D. A., GarcíaLario, P., Szczerba, R., Bobrowsky, M. 2009, A\&A, 495, L5

Pérez-Mesa, V., Zamora, O., García-Hernández D. A., Plez, B., Manchado, A., Karakas, A. I., Lugaro, M. 2017, A\&A (in press; arXiv:1706.02268)

Renzini A., Voli M., 1981, A\&A, 94, 175

Reyniers, M., Abia, C., van Winckel, H., Lloyd Evans, T., Decin, L., Eriksson, K., \& Pollard, K. R. 2007, A\&A, 461, 641

Romano D., Karakas A. I., Tosi M., Matteucci, F. 2010, A\&A, 522, A32

Sackmann I.J., Boothroyd A.I., 1992, ApJ 392, L71

Sharpee, B., Zhang, Y., Williams, R., Pellegrini, E., Cavagnolo, K., Baldwin, J. A., Phillips, M., Liu, X.-W. 2007, ApJ, 659, 1265

Smith V. V., Lambert D. L. 1989, ApJL, 345, L75

Smith V. V., Lambert D. L. 1990, ApJL, 361, L69

Smith C. L., Zijlstra A. A., Gesicki K. M., Dinerstein H. L. 2017, MNRAS, 471, 3008

Sterling, N. C., Dinerstein, H. L. 2008, ApJS, 174, 158

Sterling, N. C., Dinerstein, H. L., Kaplan, K. F.; Bautista, M. A. 2016, ApJL, 819, L9

Suárez, O., García-Lario, P., Manchado, A., Manteiga, M., 
Ulla, A., Pottasch, S. R. 2006, A\&A, 458, 173

Szczerba, R., Siódmiak, N., Stasińska, G., Borkowski, J. 2007, A\&A, 469, 799

Szczerba, R., Siódmiak, N., Stasińska, G., Borkowski, J., García-Lario, P., Suárez, O., Hajduk, M., GarcíaHernández, D. A. 2012, Proc. IAU Symp., 283, 506

Valiante R., Schneider R., Bianchi S., Andersen A., Anja C., 2009, MNRAS, 397, 1661

Valiante R., Schneider R., Salvadori S., Bianchi S. 2011, MNRAS, 416, 1916

Van Winckel, H., \& Reyniers, M. 2000, A\&A, 354, 135

Van Winckel, H. 2003, ARA\&A, 41, 391

Vassiliadis E., \& Wood P. R., 1993, ApJ, 413, 641

Vassiliadis E., \& Wood P. R., 1994, ApJS, 92, 125

Ventura P., D'Antona F., Mazzitelli I. 2000, A\&A, 363, 605

Ventura P., D'Antona F., Mazzitelli I., Gratton R. 2001, ApJL, 550, L65

Ventura P., D'Antona F., 2005, A\&A, 431, 279

Ventura, P. 2010, Light Elements in the Universe, 268, 147

Ventura P., Di Criscienzo M., Schneider R., Carini R., Valiante R., D'Antona F., Gallerani S., Maiolino R., Tornambé A., 2012a, MNRAS, 420, 1442

Ventura P., Di Criscienzo M., Schneider R., Carini R., Valiante R., D'Antona F., Gallerani S., Maiolino R., Tornambé A., 2012b, MNRAS, 424, 2345

Ventura P., Dell'Agli F., Di Criscienzo M., Schneider R., Rossi C., La Franca F., Gallerani S., Valiante R., 2014, MNRAS, 439, 977

Ventura P., Di Criscienzo M., Carini R., D'Antona F., 2013, MNRAS, 431, 3642

Ventura P., Marigo P. 2009, MNRAS, 399, L54

Ventura P., Marigo P. 2010, MNRAS, 408, 2476

Ventura, P., Zeppieri, A., Mazzitelli, I., D'Antona, F., 1998, A\&A, 334, 953

Ventura, P., Karakas, A. I., Dell'Agli, F., Boyer, M. L., García-Hernández, D. A., Di Criscienzo M., Schneider R. 2015a, MNRAS, 450, 3181

Ventura, P., Stanghellini, L., Dell'Agli, F., GarcíaHernández, D. A., Di Criscienzo M. 2015b, MNRAS, 452, 3679

Ventura P., Karakas A. I., Dell'Agli F., García-Hernández D. A., Boyer M. L., Di Criscienzo M. 2016a, MNRAS, 457, 1456

Ventura P., García-Hernández D. A., Dell'Agli, F., D'Antona, F., Mészáros, Sz., Lucatello, S., Di Criscienzo, M., Shetrone, M., Tailo, M., Tang, B., Zamora, O. 2016b, ApJL, 831, L17

Ventura, P., Stanghellini, L., Di Criscienzo M., GarcíaHernández D. A., Dell'Agli, F. 2016c, MNRAS, 460, 3940 Ventura P., Stanghellini, L., Dell'Agli, F., GarcíaHernández D. A. 2017, MNRAS (in press; arXiv:1707.08344)

Wachter A., Schröder K. P., Winters J. M., Arndt T. U., Sedlmayr E., 2002, A\&A, 384, 452

Wachter A., Winters J. M., Schröder K. P., Sedlmayr E., 2008, A\&A, 486, 497

Waters L. B. F. M., et al., 1998, A\&A, 331, L61

Weiss A., Ferguson, J. W. 2009, A\&A, 508, 1343

Yagüe, A., García-Hernández D. A., Ventura, P., Lugaro, M. 2016, Journal of Physics: Conference Series, 728, 072004

Zamora, O., García-Hernández D. A., Plez, B., Manchado,
A. 2014, A\&A, 564, L4 